\title{
ON DYNAMICS AND STABILITY OF THIN PERIODIC CYLINDRICAL SHELLS
}

\author{
BARBARA TOMCZYK
}

Received 29 December 2005; Revised 28 May 2006; Accepted 30 May 2006

The object of considerations is a thin linear-elastic cylindrical shell having a periodic structure along one direction tangent to the shell midsurface. The aim of this paper is to propose a new averaged nonasymptotic model of such shells, which makes it possible to investigate free and forced vibrations, parametric vibrations, and dynamical stability of the shells under consideration. As a tool of modeling we will apply the tolerance averaging technique. The resulting equations have constant coefficients in the periodicity direction. Moreover, in contrast with models obtained by the known asymptotic homogenization technique, the proposed one makes it possible to describe the effect of the period length on the overall shell behavior, called a length-scale effect.

Copyright (C 2006 Barbara Tomczyk. This is an open access article distributed under the Creative Commons Attribution License, which permits unrestricted use, distribution, and reproduction in any medium, provided the original work is properly cited.

\section{Introduction}

In this paper, a new nonasymptotic model for problems of dynamics and dynamical stability of thin cylindrical shells having a periodic structure (i.e., periodically varying thickness and/or periodically varying elastic and inertial properties) along one direction tangent to the shell midsurface $\mathcal{M}$ is presented. At the same time, the considered shells have slowly varying or constant structure along the direction tangent to $M$ and perpendicular to the direction of periodicity. This situation is mainly orientated towards cylindrical shells reinforced by periodically spaced dense system of ribs as shown in Figure 1.1. Shells with a periodic structure along one direction tangent to $M$ are termed uniperiodic.

The period of inhomogeneity is assumed to be very large compared with the maximum shell thickness and very small as compared to the midsurface curvature radius as well as the smallest characteristic length dimension of the shell midsurface. It means that the shells under consideration are composed of a large number of identical elements and every such element, called a periodicity cell, can be treated as a shallow shell.

It should be noted that in the general case, on the shell midsurface we do not deal with periodic but with what is called a locally periodic structure in directions tangent to 

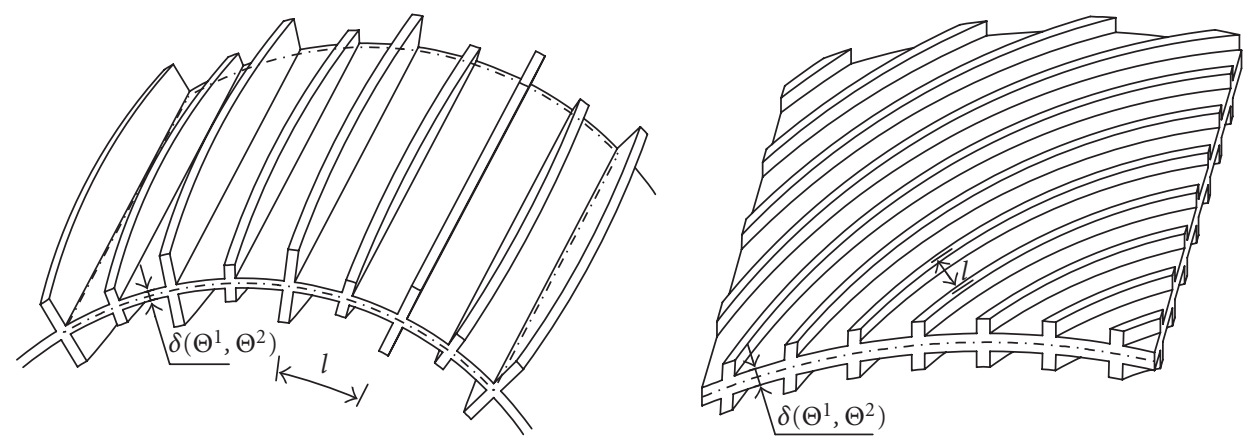

Figure 1.1. Examples of uniperiodic shells.

M. Following [20], by a locally periodic shell we mean a shell which, in the subregions of the shell midsurface $M$ much smaller than $M$, can be approximately regarded as periodic. Hence, a locally periodic shell is made of a large number of not only identical but also similar elements. However, for cylindrical shells the Gaussian curvature is equal to zero and hence on the developable cylindrical surface we can separate a cell which can be referred to as the representative cell for a whole shell midsurface. It means that on cylindrical surface we deal not with locally periodic but with aperiodic structure.

Because properties of periodic (or locally periodic) structures are described by highly oscillating, noncontinuous, periodic functions, the exact equations of the shell (plate) theory are too complicated to apply to investigations of engineering problems. That is why a lot of different approximate modeling methods for shells and plates of this kind have been proposed. Periodic cylindrical shells and plates are usually described using homogenized models derived by means of asymptotic methods. These models represent certain equivalent structures with constant or slowly varying stiffnesses and averaged mass densities (cf. [5, 9-13]). Unfortunately, in models of this kind the effect of a period length (called also the length-scale effect) on the overall shell behavior is neglected.

The periodically densely ribbed plates and shells are also modeled as homogeneous orthotropic structures (cf. $[1,7]$ ). These orthotropic models are not able to describe the length-scale effect on the overall shell (plate) behavior, being independent of the period of inhomogeneity.

In order to analyze this effect, the new averaged nonasymptotic models of thin uniperiodic cylindrical shells have been proposed in $[17,18]$. These, co called the tolerance models, have been obtained by applying the nonasymptotic tolerance averaging technique, proposed and discussed for periodic composites in the monograph [21], to the known equations of Kirchhoff-Love-type cylindrical shells (differential equations with functional highly oscillating noncontinuous periodic coefficients). These tolerance models have constant coefficients in periodicity direction and take into account the effect of a cell size on the global shell dynamics and stationary stability. This effect is described by means of certain extra unknowns called internal or fluctuation variables and by known functions which represent oscillations inside the periodicity cell, and are obtained either as approximate solutions to special eigenvalue problems for free vibrations on the separated cell with 
periodic boundary conditions or by using the finite element discretization of the cell. However, the aforementioned tolerance model of dynamic problems for periodic cylindrical shells proposed in [17], and that of stationary stability problems given in [18] cannot be used to analyze a dynamical stability and parametric vibrations of the periodic shells. That is why, in this paper the tolerance model of dynamic problems and dynamical stability problems for uniperiodic Kirchhoff-Love-type cylindrical shells, loaded by time-dependent forces tangent to the shell midsurface is derived and discussed. It means that the proposed here model can be treated as a certain generalization of the models given in $[17,18]$.

It is worth noting that the application of the tolerance averaging technique to the investigation of selected dynamic and stability problems for periodic plates can be found in many papers, that is, in $[3,15]$, where the stability of densely stiffened Kirchhoff-type plates and of Hencky-Bolle-type plates is analyzed, respectively, in [8, 14], where dynamics of Kirchhoff-type plates and of wavy-type plates is investigated, respectively, and others.

It has to be mentioned that an extremely extensive literature deals with elastic stability and dynamics of thin cylindrical shells reinforced by widely spaced stiffeners. Contrary to the shells with densely spaced ribs, which are objects of considerations in this paper, those having widely spaced stiffeners are analyzed with allowance for the discreteness in the arrangement of the ribs. It means that the dynamic and stability problems of such shells are considered within the framework of discrete models, while the dynamic and stability analysis of periodically, densely ribbed cylindrical shells investigated in this paper is carried out within continuum models. The discrete approach is in detail discussed in monographs $[2,6]$. Moreover, in the mentioned monographs it can be found an extensive review of papers and books dealing with stability and dynamic problems of widely ribbed shells as well as of densely stiffened shells treated as homogeneous orthotropic structures.

It is well known that stability problems of thin cylindrical shells being homogeneous or weakly heterogeneous have to be investigated by using the geometrically nonlinear shell theory, $[4,16,19]$. However, in the case of the highly heterogeneous structures considered here (i.e., densely ribbed shells) which are described by using continuum models, we are interested in the upper state of critical forces and hence we can use the geometrically linear stability theory for thin linear-elastic cylindrical Kirchhoff-Love-type shells.

The aim of this contribution is threefold.

(i) First, to formulate an averaged nonasymptotic model of a uniperiodic cylindrical shell, which has constant coefficients in direction of periodicity and describes the effect of a cell size on the global shell dynamics and dynamical stability. This model will be derived by using the tolerance averaging procedure proposed in [21].

(ii) Second, to derive a simplified model (called asymptotic or homogenized) in which the length-scale effect is neglected.

(iii) Third, to evaluate the effect of a cell size on the free vibrations of uniperiodic shells by using both the tolerance and homogenized models.

Basic denotations, preliminary concepts, and starting equations will be presented in Section 2. The general line of the tolerance averaging approach will be shown in Section 3. The tolerance model for problems of dynamics and dynamical stability of linear-elastic thin cylindrical shells with a periodic structure along one direction tangent to $\mathcal{M}$ and 
slowly varying or constant structure along the perpendicular direction tangent to $M$ will be proposed and discussed in Section 4. For comparison, the governing equations of a certain homogenized model will be given in Section 5. In the subsequent section, in order to evaluate the length-scale effect in dynamic problems, both the obtained tolerance and homogenized models will be applied to investigations of free vibrations in open circular cylindrical shell reinforced by ribs, which are densely and periodically spaced along the lines of principal curvature of the shell midsurface. Final remarks will be formulated in the last section.

\section{Preliminaries}

In this paper we will investigate thin linear-elastic cylindrical shells with a periodic structure along one direction tangent to $M$. Cylindrical shells of this kind will be termed uniperiodic. At the same time, the shells under consideration have slowly varying or constant structure (i.e., slowly varying or constant geometrical and/or material properties) along the direction tangent to $M$ and perpendicular to the direction of periodicity. Examples of such shells are presented in Figure 1.1.

Denote by $\Omega \subset R^{2}$ a regular region of points $\Theta \equiv\left(\Theta^{1}, \Theta^{2}\right)$ on the $O \Theta^{1} \Theta^{2}$-plane, $\Theta^{1}, \Theta^{2}$ being the Cartesian orthogonal coordinates on this plane, and let $E^{3}$ be the physical space parametrized by the Cartesian orthogonal coordinate system $O x^{1} x^{2} x^{3}$. Let us introduce the orthogonal parametric representation of the undeformed smooth cylindrical shell midsurface $\mathcal{M}$ by means of $\mathcal{M}:=\left\{\mathbf{x} \equiv\left(x^{1}, x^{2}, x^{3}\right) \in E^{3}: \mathbf{x}=\mathbf{x}\left(\Theta^{1}, \Theta^{2}\right), \boldsymbol{\Theta} \in \Omega\right\}$, where $\mathbf{x}\left(\Theta^{1}, \Theta^{2}\right)$ is a position vector of a point on $\mathcal{M}$ having coordinates $\Theta^{1}, \Theta^{2}$.

Throughout the paper indices $\alpha, \beta, \ldots$ run over 1,2 and are related to the midsurface parameters $\Theta^{1}, \Theta^{2}$; indices $A, B, \ldots$ run over $1,2, \ldots, N$, summation convention holds for all aforesaid indices.

To every point $\mathbf{x}=\mathbf{x}(\boldsymbol{\Theta}), \boldsymbol{\Theta} \in \Omega$, we assign a covariant base vectors $\mathbf{a}_{\alpha}=\mathbf{x},{ }_{\alpha}$ and covariant midsurface first and second metric tensors denoted by $a_{\alpha \beta}, b_{\alpha \beta}$, respectively, which are given as follows: $a_{\alpha \beta}=\mathbf{a}_{\alpha} \cdot \mathbf{a}_{\beta}, b_{\alpha \beta}=\mathbf{n} \cdot \mathbf{a}_{\alpha, \beta}$, where $\mathbf{n}$ is a unit vector normal to $\mathcal{M}$.

Let $\delta(\Theta)$ stand for the shell thickness. We also define $t$ as the time coordinate.

Taking into account that coordinate lines $\Theta^{2}=$ const are parallel on the $O \Theta^{1} \Theta^{2}$-plane and that $\Theta^{2}$ is an arc coordinate on $M$, we define $l$ as the period of shell structure in $\Theta^{2}$ direction. The period $l$ is assumed to be sufficiently large compared with the maximum shell thickness and sufficiently small as compared with the midsurface curvature radius $R$ as well as the characteristic length dimension $L$ of the shell midsurface along the direction of shell periodicity, that is, $\sup \delta(\cdot) \ll l \ll \min \{R, L\}$.

We will denote by $\Lambda \equiv\{0\} \times(-l / 2, l / 2)$ the straight line segment on the $O \Theta^{1} \Theta^{2}$-plane along the $O \Theta^{2}$-axis direction, which can be taken as a representative cell of the periodic shell structure (the periodicity cell). To every $\Theta \in \Omega$ an arbitrary cell on $O \Theta^{1} \Theta^{2}$-plane will be defined by means of $\Lambda(\boldsymbol{\Theta}) \equiv \boldsymbol{\Theta}+\Lambda, \boldsymbol{\Theta} \in \Omega_{\Lambda}, \Omega_{\Lambda}:=\{\boldsymbol{\Theta} \in \Omega: \Lambda(\boldsymbol{\Theta}) \subset \Omega\}$, where the point $\Theta \in \Omega_{\Lambda}$ is a center of a cell $\Lambda(\Theta)$ and $\Omega_{\Lambda}$ is a set of all the cell centers which are inside $\Omega$. 
A function $f(\Theta)$ defined on $\Omega_{\Lambda}$ will be called $\Lambda$-periodic if for arbitrary but fixed $\Theta^{1}$ and arbitrary $\Theta^{2}, \Theta^{2} \pm l$ it satisfies the condition $f\left(\Theta^{1}, \Theta^{2}\right)=f\left(\Theta^{1}, \Theta^{2} \pm l\right)$ in the whole domain of its definition and it is not constant.

It is assumed that the cylindrical shell thickness as well as its elastic and inertial properties are $\Lambda$-periodic functions of $\Theta^{2}$ and slowly varying or constant functions of $\Theta^{1}$. Shells like that are called uniperiodic.

The above periodic heterogeneities can be also interpreted as those caused by a periodically spaced dense system of ribs, as shown in Figure 1.1.

For an arbitrary integrable function $\varphi(\cdot)$ defined on $\Omega$, following [21], we define the averaging operation, given by

$$
\langle\varphi\rangle(\Theta) \equiv \frac{1}{l} \int_{\Lambda(\Theta)} \varphi\left(\Theta^{1}, \Psi^{2}\right) d \Psi^{2}, \quad\left(\Theta^{1}, \Psi^{2}\right) \in \Lambda(\Theta), \quad \Theta=\left(\Theta^{1}, \Theta^{2}\right) \in \Omega_{\Lambda} .
$$

For a function $\varphi$, which is $\Lambda$-periodic in $\Theta^{2}$, formula (2.1) leads to $\langle\varphi\rangle\left(\Theta^{1}\right)$. If the function $\varphi$ is $\Lambda$-periodic in $\Theta^{2}$ and is independent of $\Theta^{1}$, its averaged value obtained from (2.1) is constant.

The denotation $\cong$ is used for a tolerance relation (cf. Section 3 ) and $\simeq$ denotes an approximation due to the truncation of the infinite series (cf. Section 4).

Our considerations will be based on the simplified linear Kirchhoff-Love second-order theory of thin elastic shells in which terms depending on the second metric tensor of $M$ are neglected in the formulae for curvature changes. Below, we quote the general formulations of the theory under consideration.

2.1. The Kirchhoff-Love shell equations. Let $u_{\alpha}(\boldsymbol{\Theta}, t), w(\boldsymbol{\Theta}, t)$ stand for the midsurface shell displacements in directions tangent and normal to $\mathcal{M}$, respectively. We denote by $\varepsilon_{\alpha \beta}(\boldsymbol{\Theta}, t), \kappa_{\alpha \beta}(\boldsymbol{\Theta}, t)$ the membrane and curvature strain tensors and by $n^{\alpha \beta}(\boldsymbol{\Theta}, t), m^{\alpha \beta}(\boldsymbol{\Theta}, t)$ the stress resultants and stress couples, respectively. The elastic properties of the shell are described by $2 D$-shell stiffness tensors $D^{\alpha \beta \gamma \delta}(\boldsymbol{\Theta}), B^{\alpha \beta \gamma \delta}(\boldsymbol{\Theta})$. Let $\mu(\boldsymbol{\Theta})$ stand for the shell mass density per midsurface unit area. Let $f_{\alpha}(\boldsymbol{\Theta}, t), f(\boldsymbol{\Theta}, t)$ be external force components per midsurface unit area, respectively, tangent and normal to $\mathcal{M}$. We denote by $\bar{N}^{\alpha \beta}(t)$ the time-dependent compressive membrane forces in the shell midsurface.

Functions $\mu(\boldsymbol{\Theta}), D^{\alpha \beta \gamma \delta}(\boldsymbol{\Theta}), B^{\alpha \beta \gamma \delta}(\boldsymbol{\Theta})$, and $\delta(\boldsymbol{\Theta})$ are $\Lambda$-periodic functions of $\Theta^{2}$ and are assumed to be slowly varying functions of $\Theta^{1}$.

The equations of a shell theory under consideration consist of

(i) the strain-displacement equations

$$
\varepsilon_{\gamma \delta}=u_{(\gamma, \delta)}-b_{\gamma \delta} w, \quad \kappa_{\gamma \delta}=-w_{, \gamma \delta},
$$

(ii) the stress-strain relations

$$
n^{\alpha \beta}=D^{\alpha \beta \chi \delta} \varepsilon_{\gamma \delta}, \quad m^{\alpha \beta}=B^{\alpha \beta \chi \delta} \kappa_{\gamma \delta},
$$

(iii) the equations of motions

$$
n_{, \alpha}^{\alpha \beta}-\mu a^{\alpha \beta} \ddot{u}_{\alpha}+f^{\beta}=0, \quad m_{, \alpha \beta}^{\alpha \beta}+b_{\alpha \beta} n^{\alpha \beta}-\bar{N}^{\alpha \beta} w_{, \alpha \beta}-\mu \ddot{w}+f=0 .
$$


In the above equations the displacements $u_{\alpha}(\boldsymbol{\Theta}, t)$ and $w(\boldsymbol{\Theta}, t), \boldsymbol{\Theta} \in \Omega$, are the basic unknowns.

For uniperiodic shells, $\mu(\boldsymbol{\Theta}), D^{\alpha \beta \gamma \delta}(\boldsymbol{\Theta})$, and $B^{\alpha \beta \gamma \delta}(\boldsymbol{\Theta}), \boldsymbol{\Theta} \in \Omega$, are noncontinuous highly oscillating $\Lambda$-periodic functions; that is why (2.2)-(2.4) cannot be directly applied to the numerical analysis of special problems. From (2.2)-(2.4) an averaged model of uniperiodic cylindrical shells under consideration having coefficients, which are independent of $\Theta^{2}$-midsurface parameter and are slowly varying (or constant) functions of $\Theta^{1}$ as well as describing the cell size effect on the global shell behavior, will be derived. In order to derive it, the tolerance averaging procedure given in [21] will be applied. To make the analysis more clear, in the next section we will outline the basic concepts and the main assumptions of this approach, following the monograph [21].

\section{Modeling concepts and assumptions}

Following the monograph [21], we outline below the basic concepts and assumptions which will be used in the course of modeling procedure.

3.1. Basic concepts. The fundamental concepts of the tolerance averaging approach are those of a certain tolerance system, slowly varying functions, periodic-like functions, and periodic-like oscillating functions. These functions will be defined with respect to the $\Lambda$ periodic shell structure defined in the foregoing section.

By a tolerance system we will mean a pair $T=(\mathscr{F}, \varepsilon(\cdot))$, where $\mathscr{F}$ is a set of real-valued bounded functions $F(\cdot)$ defined on $\bar{\Omega}$ and their derivatives (including also time derivatives), which represent the unknowns in the problem under consideration (such as unknown shell displacements tangent and normal to $\mathcal{M}$ ) and for which the tolerance parameters $\varepsilon_{F}$ being positive real numbers and determining the admissible accuracy related to computations of values of $F(\cdot)$ are given; by $\varepsilon$ is denoted the mapping $\mathscr{F} \in F \rightarrow \varepsilon_{F}$.

A continuous bounded differentiable function $F(\Theta, t)$ defined on $\bar{\Omega}$ is called $\Lambda$-slowly varying with respect to the cell $\Lambda$ and the tolerance system $T, F \in \operatorname{SV}_{\Lambda}(T)$, if roughly speaking, can be treated (together with its derivatives) as constant on an arbitrary periodicity cell $\Lambda$.

The continuous function $\varphi(\cdot)$ defined on $\bar{\Omega}$ will be termed $a \Lambda$-periodic-like function, $\varphi(\cdot) \in \mathrm{PL}_{\Lambda}(T)$, with respect to the cell $\Lambda$ and the tolerance system $T$, if for every $\Theta=$ $\left(\Theta^{1}, \Theta^{2}\right) \in \Omega_{\Lambda}$, there exists a continuous $\Lambda$-periodic function $\varphi_{\Theta}(\cdot)$ such that (for all $\left.\boldsymbol{\Psi}=\left(\Theta^{1}, \Psi^{2}\right)\right)\left[\|\boldsymbol{\Theta}-\boldsymbol{\Psi}\| \leq l \Rightarrow \varphi(\boldsymbol{\Psi}) \cong \varphi_{\Theta}(\boldsymbol{\Psi})\right], \boldsymbol{\Psi} \in \Lambda(\boldsymbol{\Theta})$, and the similar conditions are also fulfilled by all its derivatives. It means that the values of a periodic-like function $\varphi(\cdot)$ in an arbitrary cell $\Lambda(\Theta), \Theta \in \Omega_{\Lambda}$, can be approximated, with sufficient accuracy, by the corresponding values of a certain $\Lambda$-periodic function $\varphi_{\Theta}(\cdot)$. The function $\varphi_{\Theta}(\cdot)$ will be referred to as a $\Lambda$-periodic approximation of $\varphi(\cdot)$ on $\Lambda(\boldsymbol{\Theta})$.

Let $\mu(\cdot)$ be a positive value $\Lambda$-periodic function. The periodic-like function $\varphi$ is called $\Lambda$ oscillating (with the weight $\mu$ ), $\varphi(\cdot) \in \operatorname{PL}_{\Lambda}^{\mu}(T)$, provided that the condition $\langle\mu \varphi\rangle(\Theta) \cong 0$ holds for every $\Theta \in \Omega_{\Lambda}$. In the special case $\mu=$ const the oscillating periodic-like functions satisfy condition $\langle\varphi\rangle(\boldsymbol{\Theta}) \cong 0, \boldsymbol{\Theta} \in \Omega$; in this case we will write $\varphi \in \operatorname{PL}_{\Lambda}^{1}(T)$. 
3.2. Modelling assumptions. The tolerance averaging technique is based on two modeling assumptions. The first of them is strictly related to the concept of $\Lambda$-slowly varying and $\Lambda$-periodic-like functions.

Tolerance averaging assumption. If $F \in \operatorname{SV}_{\Lambda}(T), \varphi(\cdot) \in \mathrm{PL}_{\Lambda}(T)$, and $\varphi_{\Theta}(\cdot)$ is a $\Lambda$-periodic approximation of $\varphi(\cdot)$ on $\Lambda(\Theta)$, then for every $\Lambda$-periodic bounded function $f(\cdot)$ and every continuous $\Lambda$-periodic differentiable function $h(\cdot)$, such that $\sup \left\{\left|h\left(\Psi^{1}, \Psi^{2}\right)\right|\right.$, $\left.\left(\Psi^{1}, \Psi^{2}\right) \in \Lambda\right\} \leq l$, the following tolerance averaging relations determined by the pertinent tolerance parameters hold for every $\Theta \in \Omega_{\Lambda}$ :

$$
\begin{aligned}
(\mathbf{T} 1)\langle f F\rangle(\boldsymbol{\Theta}) \cong\langle f\rangle(\boldsymbol{\Theta}) F(\boldsymbol{\Theta}), & (\mathbf{T} 2)\left\langle f(h F)_{, 2}\right\rangle(\boldsymbol{\Theta}) \cong\langle f F h, 2\rangle(\boldsymbol{\Theta}), \\
(\mathbf{T} 3)\langle f \varphi\rangle(\boldsymbol{\Theta}) \cong\left\langle f \varphi_{\Theta}\right\rangle(\boldsymbol{\Theta}), & (\mathbf{T} 4)\left\langle h(f \varphi)_{, 2}\right\rangle(\boldsymbol{\Theta}) \cong-\left\langle f \varphi h_{, 2}\right\rangle(\boldsymbol{\Theta}) .
\end{aligned}
$$

It means that in the course of averaging the left-hand sides of formulae (T1)-(T4) can be approximated by their right-hand sides, respectively.

The second modeling assumption is based on heuristic premises.

Conformability assumption. In every periodic solid the displacement fields have to conform to the periodic structure of this solid. It means that the displacement fields are periodic-like functions and hence can be represented by a sum of averaged displacements, which are slowly varying (with respect to the cell and tolerance system), and by highly oscillating periodic-like disturbances, caused by the periodic structure of the solid.

The aforementioned conformability assumption together with the tolerance averaging assumption constitute the foundations of the tolerance averaging technique. Using this technique the tolerance model of uniperiodic cylindrical shells for problems of dynamics and dynamical stability will be derived in the subsequent section.

\section{The tolerance model}

4.1. Modeling procedure. Let us assume that there is a certain tolerance system $T=$ $(\mathscr{F}, \varepsilon(\cdot))$, where the set $F$ consists of the unknown shell displacements tangent and normal to $\mathcal{M}$ and their derivatives.

The tolerance averaging approach to (2.2)-(2.4) will be realized in five steps.

Step 1. From the conformability assumption, it follows that the unknown shell displacements $u_{\alpha}(\boldsymbol{\Theta}, t), w(\boldsymbol{\Theta}, t)$ in (2.2)-(2.4) have to satisfy the conditions $u_{\alpha}(\boldsymbol{\Theta}, t) \in \operatorname{PL}_{\Lambda}(T)$, $w(\boldsymbol{\Theta}, t) \in \mathrm{PL}_{\Lambda}(T)$ and hence can be decomposed into

$$
u_{\alpha}(\boldsymbol{\Theta}, t)=U_{\alpha}(\boldsymbol{\Theta}, t)+d_{\alpha}(\boldsymbol{\Theta}, t), \quad w(\boldsymbol{\Theta}, t)=W(\boldsymbol{\Theta}, t)+p(\boldsymbol{\Theta}, t),
$$

where $U_{\alpha}(\boldsymbol{\Theta}, t), W(\boldsymbol{\Theta}, t) \in \mathrm{SV}_{\Lambda}(T)$ are the averaged parts of displacements $u_{\alpha}(\boldsymbol{\Theta}, t), w(\boldsymbol{\Theta}$, $t)$, respectively, called macrodisplacements and defined by $U_{\alpha}(\cdot, t) \equiv\langle\mu\rangle^{-1}\left(\Theta^{1}\right)\left\langle\mu u_{\alpha}\right\rangle(\cdot, t)$, $W(\cdot, t) \equiv\langle\mu\rangle^{-1}\left(\Theta^{1}\right)\langle\mu w\rangle(\cdot, t)$ (cf. [21]) and $d_{\alpha}(\cdot, t), p(\cdot, t) \in \mathrm{PL}_{\Lambda}^{\mu}(T)$ are the fluctuating parts of displacements $u_{\alpha}(\boldsymbol{\Theta}, t), w(\boldsymbol{\Theta}, t)$, respectively, such that $\left\langle\mu d_{\alpha}(\boldsymbol{\Theta}, t)\right\rangle=\langle\mu p(\boldsymbol{\Theta}$, $t)\rangle=0$. 
Step 2. Substituting the right-hand side of (4.1) into (2.4) and after the tolerance averaging of the resulting equations, we arrive at the following equations:

$$
\begin{aligned}
& {\left[\left\langle D^{\alpha \beta \gamma \delta}\right\rangle\left(\Theta^{1}\right)\left(U_{\gamma, \delta}-b_{\gamma \delta} W\right)+\left\langle D^{\alpha \beta \gamma \delta} d_{\gamma, \delta}\right\rangle(\Theta, t)-b_{\gamma \delta}\left\langle D^{\alpha \beta \gamma \delta} p\right\rangle(\Theta, t)\right]_{, \alpha}} \\
& \quad-\langle\mu\rangle\left(\Theta^{1}\right) a^{\alpha \beta} \ddot{U}_{\alpha}=-\left\langle f^{\beta}\right\rangle(\Theta, t), \\
& {\left[\left\langle B^{\alpha \beta \gamma \delta}\right\rangle\left(\Theta^{1}\right) W_{, \gamma \delta}+\left\langle B^{\alpha \beta \gamma \delta} p_{, \gamma \delta}\right\rangle(\Theta, t)\right]_{, \alpha \beta}} \\
& \quad-b_{\alpha \beta}\left[\left\langle D^{\alpha \beta \gamma \delta}\right\rangle\left(\Theta^{1}\right)\left(U_{\gamma, \delta}-b_{\gamma \delta} W\right)+\left\langle D^{\alpha \beta \gamma \delta} d_{\gamma, \delta}\right\rangle(\Theta, t)-b_{\gamma \delta}\left\langle D^{\alpha \beta \gamma \delta} p\right\rangle\right] \\
& \quad+\bar{N}^{\alpha \beta} W_{\alpha \beta}+\langle\mu\rangle\left(\Theta^{1}\right) \ddot{W}=\langle f\rangle(\Theta, t),
\end{aligned}
$$

which must hold for every $\Theta \in \Omega_{\Lambda}$ and every time $t$.

The above averaging implies the condition $\left\langle f^{\beta}\right\rangle(\boldsymbol{\Theta}, t),\langle f\rangle(\boldsymbol{\Theta}, t) \in \mathrm{SV}_{\Lambda}(T)$. This situation takes place if the shell external loadings satisfy the condition $f^{\beta}(\boldsymbol{\Theta}, t), f(\boldsymbol{\Theta}, t) \in$ $\operatorname{PL}_{\Lambda}(T)$. Subsequently we will use the decomposition $f^{\beta}(\cdot, t)=f_{0}^{\beta}(\cdot, t)+\tilde{f}^{\beta}(\cdot, t), f(\cdot, t)=$ $f_{0}(\cdot, t)+\tilde{f}(\cdot, t)$, where $f_{0}^{\beta}(\cdot, t), f_{0}(\cdot, t) \in \operatorname{SV}_{\Lambda}(T), \tilde{f}^{\beta}(\cdot, t), \tilde{f}(\cdot, t) \in \operatorname{PL}_{\Lambda}^{1}(T)$, and $\left\langle\tilde{f}^{\beta}\right\rangle(\boldsymbol{\Theta}$, $t)=\langle\tilde{f}\rangle(\boldsymbol{\Theta}, t)=0$.

Step 3. Multiplying $(2.4)_{1}$ and $(2.4)_{2}$ by arbitrary $\Lambda$-periodic test functions $d^{*}, p^{*}$, respectively, such that $\left\langle\mu d^{*}\right\rangle=\left\langle\mu p^{*}\right\rangle=0$, integrating these equations over $\Lambda(\Theta), \Theta \in \Omega_{\Lambda}$, and using the tolerance averaging assumption, as well as denoting by $\tilde{d}_{\alpha}, \tilde{p}$ the $\Lambda$-periodic approximations of $d_{\alpha}, p$, respectively, on $\Lambda(\Theta)$, we obtain the periodic problem on $\Lambda(\Theta)$ for functions $\tilde{d}_{\alpha}\left(\Theta^{1}, \Psi^{2}, t\right), \tilde{p}\left(\Theta^{1}, \Psi^{2}, t\right),\left(\Theta^{1}, \Psi^{2}\right) \in \Lambda(\Theta)=\Lambda\left(\Theta^{1}, \Theta^{2}\right)$, given by the following variational conditions:

$$
\begin{aligned}
&-\left\langle d_{, 2}^{*} D^{2 \beta \gamma \delta} \tilde{d}_{\gamma, \delta}\right\rangle+\left\langle d^{*}\left(D^{1 \beta \gamma \delta} \tilde{d}_{\gamma, \delta}\right)_{, 1}\right\rangle-b_{\gamma \delta}\left[-\left\langle d_{, 2}^{*} D^{2 \beta \gamma \delta} \tilde{p}\right\rangle+\left\langle d^{*}\left(D^{1 \beta \gamma \delta \tilde{p}}\right)_{, 1}\right\rangle\right]-\left\langle d^{*} \mu \ddot{\widetilde{d}}\right\rangle a^{\alpha \beta} \\
&=-\left\langle d^{*} f^{\beta}\right\rangle+\left\langle d_{, \alpha}^{*} D^{\alpha \beta \gamma \delta}\right\rangle\left(U_{\gamma, \delta}-b_{\gamma \delta} W\right)-\left[\left\langle d^{*} D^{1 \beta \gamma \delta}\right\rangle\left(U_{\gamma, \delta}-b_{\gamma \delta} W\right)\right]_{, 1}, \\
&\left\langle p_{, 22}^{*} B^{22 \gamma \delta} \tilde{p}_{, \gamma \delta}\right\rangle-2\left\langle p_{, 2}^{*}\left(B^{21 \gamma \delta} \tilde{p}_{, \gamma \delta}\right)_{, 1}\right\rangle+\left\langle p^{*}\left(B^{11 \gamma \delta} \tilde{p}, \gamma \delta_{, 11}\right\rangle\right. \\
& \quad-b_{\alpha \beta}\left[\left\langle p^{*} D^{\alpha \beta \gamma \delta} \tilde{d}_{\gamma, \delta}\right\rangle-b_{\gamma \delta}\left\langle p^{*} D^{\alpha \beta \gamma \delta} \tilde{p}\right\rangle\right]+\left\langle p^{*} \mu \ddot{\tilde{p}}\right\rangle+\bar{N}^{11}\left\langle p^{*} \tilde{p}_{, 11}\right\rangle \\
&+2 \bar{N}^{12}\left\langle p^{*} \tilde{p}_{, 12}\right\rangle+\bar{N}^{22}\left\langle p^{*} \tilde{p}_{, 22}\right\rangle \\
&=\left\langle p^{*} f\right\rangle+b_{\alpha \beta}\left\langle p^{*} D^{\alpha \beta \gamma \delta}\right\rangle\left(U_{\gamma, \delta}-b_{\gamma \delta} W\right)-\left\langle p_{, 22}^{*} B^{22 \lambda \delta}\right\rangle W_{, \gamma \delta} \\
&+ 2\left[\left(\left\langle p_{, 2}^{*} B^{21 \gamma \delta}\right\rangle_{, 1}-\left\langle p_{, 21}^{*} B^{21 \gamma \delta}\right\rangle\right) W_{, \gamma \delta}+\left\langle p_{, 2}^{*} B^{21 \gamma \delta}\right\rangle W_{, \gamma \delta 1}\right] \\
&-\left\{\left[\left(\left\langle p^{*} B^{11 \gamma \delta}\right\rangle_{, 1}-2\left\langle p_{, 1}^{*} B^{11 \lambda \delta}\right\rangle\right)_{, 1}+\left\langle p_{, 11}^{*} B^{11 \gamma \delta}\right\rangle\right] W_{, \gamma \delta}\right. \\
&\left.+2\left(\left\langle p^{*} B^{11 \gamma \delta}\right\rangle_{, 1}-\left\langle p_{, 1}^{*} B^{11 \gamma \delta}\right\rangle\right) W_{, \gamma \delta 1}+\left\langle p^{*} B^{11 \gamma \delta}\right\rangle W_{, \gamma \delta 11}\right\} .
\end{aligned}
$$


Conditions (4.3) $)_{1}$ and $(4.3)_{2}$ must hold for every $\Lambda$-periodic test function $d^{*}$ and for every $\Lambda$-periodic test function $p^{*}$, respectively.

Equations (4.2), (4.3) represent the basis for obtaining the tolerance model of thin linear elastic uniperiodic cylindrical shells, which makes it possible to investigate free and forced vibrations, parametric vibrations, dynamical stability, and stationary stability (after neglecting the inertial forces and time coordinate).

Step 4. In order to obtain solution to the periodic problem on $\Lambda(\Theta)$, given by the variational equations (4.3), we can apply the known orthogonalization method. Hence, for arbitrary $\Theta^{1}$ and $\left(\Theta^{1}, \Psi^{2}\right) \in \Lambda(\Theta), \Theta=\left(\Theta^{1}, \Theta^{2}\right) \in \Omega_{\Lambda}$, we can look for solutions to the periodic problem (4.3) in the form of the finite series

$$
\begin{gathered}
\tilde{d}_{\alpha}\left(\Theta^{1}, \Psi^{2}, t\right)=h^{A}\left(\Theta^{1}, \Psi^{2}\right) Q_{\alpha}^{A}\left(\Theta^{1}, \Theta^{2}, t\right), \\
\tilde{p}\left(\Theta^{1}, \Psi^{2}, t\right)=g^{A}\left(\Theta^{1}, \Psi^{2}\right) V^{A}\left(\Theta^{1}, \Theta^{2}, t\right), \quad A=1,2, \ldots, N,
\end{gathered}
$$

in which the choice of a number $N$ of terms in the finite sums determines different degrees of approximations and where $Q_{\alpha}^{A}\left(\Theta^{1}, \Theta^{2}, t\right), V^{A}\left(\Theta^{1}, \Theta^{2}, t\right)$ are new unknowns called fluctuation variables, being $\Lambda$-slowly varying functions in $\Theta^{2}$, that is, $Q_{\alpha}^{A}, V^{A} \in \mathrm{SV}_{\Lambda}(T)$. Moreover, $h^{A}\left(\Theta^{1}, \Psi^{2}\right), g^{A}\left(\Theta^{1}, \Psi^{2}\right), A=1, \ldots, N$, are known in every problem under consideration, linear-independent, $l$-periodic functions such that $h^{A}, l h_{, 2}^{A}, l^{-1} g^{A}, g_{2,2}^{A}, g_{, 22}^{A} \in$ $O(l)$ and $\max \left|h^{A}\left(\Theta^{1}, \Psi^{2}\right)\right| \leq l, \max \left|g^{A}\left(\Theta^{1}, \Psi^{2}\right)\right| \leq l^{2}$ as well as $\left\langle\mu h^{A}\right\rangle\left(\Theta^{1}\right)=\left\langle\mu g^{A}\right\rangle\left(\Theta^{1}\right)=0$ for every $A$ and $\left\langle\mu h^{A} h^{B}\right\rangle\left(\Theta^{1}\right)=\left\langle\mu g^{A} g^{B}\right\rangle\left(\Theta^{1}\right)=0$ for every $A \neq B$.

Functions $h^{A}\left(\Theta^{1}, \Psi^{2}\right), g^{A}\left(\Theta^{1}, \Psi^{2}\right), A=1,2, \ldots N$, in (4.4) can be derived from the periodic finite element method discretization of the cell and hence will be referred to as the shape functions. It can be observed that in many cases this discretization of the cell requires a large number of finite elements and consequently the number $N$ of extra unknowns $Q_{\alpha}^{A}, V^{A}$ in (4.4) is also large.

The functions $h^{A}\left(\Theta^{1}, \Psi^{2}\right), g^{A}\left(\Theta^{1}, \Psi^{2}\right), A=1, \ldots, N$, can also be obtained as exact or approximate solutions to certain periodic eigenvalue problems on the cell describing free periodic vibrations of the cell. It means that the functions $h^{A}, g^{A}$ represent the expected forms of free periodic vibration modes of an arbitrary cell and hence are referred to as the mode-shape functions. Following [17], this periodic eigenvalue problem of finding $\Lambda$-periodic eigenfunctions $h_{\alpha}\left(\Theta^{1}, \Psi^{2}\right), g\left(\Theta^{1}, \Psi^{2}\right),\left(\Theta^{1}, \Psi^{2}\right) \in \Lambda(\Theta), \Theta=\left(\Theta^{1}, \Theta^{2}\right) \in \Omega_{\Lambda}$, is given by the equations

$$
\begin{gathered}
{\left[D^{2 \beta \gamma 2}\left(\Theta^{1}, \Psi^{2}\right) h_{\gamma, 2}\left(\Theta^{1}, \Psi^{2}\right)\right]_{, 2}+\mu\left(\Theta^{1}, \Psi^{2}\right)\left[\omega\left(\Theta^{1}\right)\right]^{2} a^{\alpha \beta} h_{\alpha}\left(\Theta^{1}, \Psi^{2}\right)=0} \\
{\left[B^{2222}\left(\Theta^{1}, \Psi^{2}\right) g_{, 22}\left(\Theta^{1}, \Psi^{2}\right)\right]_{, 22}-\mu\left(\Theta^{1}, \Psi^{2}\right)\left[\omega\left(\Theta^{1}\right)\right]^{2} g\left(\Theta^{1}, \Psi^{2}\right)=0}
\end{gathered}
$$

and by the periodic boundary conditions on the cell $\Lambda(\boldsymbol{\Theta})$ together with the continuity conditions inside $\Lambda(\Theta)$; by $\omega\left(\Theta^{1}\right)$ we have denoted the free vibration frequency. By averaging the above equations over $\Lambda(\Theta)$ we obtain $\left\langle\mu h_{\alpha}\right\rangle\left(\Theta^{1}\right)=\langle\mu g\rangle\left(\Theta^{1}\right)=0$.

Thus, $\left[h_{\alpha}^{1}\left(\Theta^{1}, \Psi^{2}\right), g^{1}\left(\Theta^{1}, \Psi^{2}\right)\right],\left[h_{\alpha}^{2}\left(\Theta^{1}, \Psi^{2}\right), g^{2}\left(\Theta^{1}, \Psi^{2}\right)\right], \ldots$ is a sequence of eigenfunctions related to the sequence of eigenvalues $\left[\omega_{\alpha}^{2}, \omega^{2}\right]_{1},\left[\omega_{\alpha}^{2}, \omega^{2}\right]_{2}, \ldots$ In the modeling 
procedure this sequence is restricted to the $N \geq 1$ eigenfunctions. Moreover, in most problems the analysis will be restricted to the simplest case $N=1$ in which we take into account only the lowest natural vibration modes (in directions tangent and normal to $M$ ) related to $(4.5)$.

In this paper it is assumed that $h_{1}^{A}=h_{2}^{A}$ and hence we denote $h^{A} \equiv h_{1}^{A}=h_{2}^{A}$.

Step 5. Substituting the right-hand sides of (4.4) into (4.2) and (4.3) and setting $d^{*}=$ $h^{A}\left(\Theta^{1}, \Psi^{2}\right), p^{*}=g^{A}\left(\Theta^{1}, \Psi^{2}\right), A=1,2, \ldots, N$, in (4.3), on the basis of the tolerance averaging assumption we arrive at the tolerance model of uniperiodic cylindrical shells for problems of dynamics and dynamical stability. In the next subsection the equations of this model will be given and discussed.

4.2. Governing equations of the nonasymptotic model. In the previous subsection, applying the tolerance averaging of Kirchhoff-Love second-order shell equations we have arrived at the tolerance model of dynamic and stability problems for shells having a periodic structure along one direction tangent to the shell midsurface.

Under the following extra denotations:

$$
\begin{aligned}
& \tilde{D}^{\alpha \beta \gamma \delta} \equiv\left\langle D^{\alpha \beta \gamma \delta}\right\rangle, \quad D^{A \alpha \beta \gamma} \equiv\left\langle D^{\alpha \beta \gamma \delta} h_{, \delta}^{A}\right\rangle, \quad \bar{D}^{A \alpha \beta \gamma} \equiv l^{-1}\left\langle D^{\alpha \beta \gamma 1} h^{A}\right\rangle, \\
& L^{A \alpha \beta} \equiv l^{-2} b_{\gamma \delta}\left\langle D^{\alpha \beta \gamma \delta} g^{A}\right\rangle, \quad \widetilde{B}^{\alpha \beta \gamma \delta} \equiv\left\langle B^{\alpha \beta \gamma \delta}\right\rangle, \quad K^{A \alpha \beta} \equiv\left\langle B^{\alpha \beta \gamma \delta} g_{, \gamma \delta}^{A}\right\rangle, \\
& \bar{K}^{A \alpha \beta} \equiv l^{-1}\left\langle B^{\alpha \beta 1} g_{, \delta}^{A}\right\rangle, \quad \breve{K}^{A \alpha \beta} \equiv l^{-2}\left\langle B^{\alpha \beta 11} g^{A}\right\rangle, \\
& C^{A B \beta \gamma} \equiv\left\langle D^{\alpha \beta \gamma \delta} h_{, \alpha}^{A} h_{, \delta}^{B}\right\rangle, \quad \bar{C}^{A B \beta \gamma} \equiv l^{-1}\left\langle D^{\alpha \beta \gamma 1} h_{, \alpha}^{A} h^{B}\right\rangle, \\
& F^{A B \beta} \equiv l^{-2} b_{\gamma \delta}\left\langle D^{\alpha \beta \gamma \delta} h_{, \alpha}^{A} g^{B}\right\rangle, \quad \widetilde{C}^{A B \beta \gamma} \equiv l^{-2}\left\langle D^{1 \beta \gamma 1} h^{A} h^{B}\right\rangle, \\
& \bar{F}^{A B \beta} \equiv l^{-3} b_{\gamma \delta}\left\langle D^{1 \beta \gamma \delta} h^{A} g^{B}\right\rangle, \quad S^{A B} \equiv\left\langle B^{\alpha \beta \gamma \delta} g_{, \alpha \beta}^{A} g_{, \gamma \delta}^{B}\right\rangle, \\
& \bar{L}^{A B} \equiv l^{-4} b_{\alpha \beta} b_{\gamma \delta}\left\langle D^{\alpha \beta \gamma \delta} g^{A} g^{B}\right\rangle, \quad \stackrel{-A B}{R} \equiv l^{-1}\left\langle B^{1 \beta \gamma \delta} g_{, \beta}^{A} g_{, \gamma \delta}^{B}\right\rangle, \\
& \widetilde{R}^{A B} \equiv l^{-2}\left\langle B^{11 \gamma \delta} g_{, \gamma \delta}^{A} g^{B}\right\rangle, \quad \bar{R}^{A B} \equiv l^{-3}\left\langle B^{1 \beta 11} g_{, \beta}^{A} g^{B}\right\rangle, \\
& \hat{R}^{A B} \equiv l^{-4}\left\langle B^{1111} g^{A} g^{B}\right\rangle, \quad \tilde{S}^{A B} \equiv l^{-2}\left\langle B^{1 \gamma 1 \delta} g_{, \gamma}^{A} g_{, \delta}^{B}\right\rangle,
\end{aligned}
$$

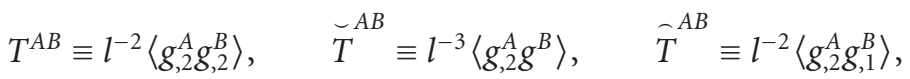

$$
\begin{aligned}
& \widetilde{\widetilde{T}}^{A B} \equiv l^{-2}\left\langle g_{, 11}^{A} g^{B}\right\rangle, \quad \tilde{T}^{A B} \equiv l^{-3}\left\langle g_{, 1}^{A} g^{B}\right\rangle, \quad \bar{T}^{A B} \equiv l^{-4}\left\langle g^{A} g^{B}\right\rangle, \\
& \tilde{\mu} \equiv\langle\mu\rangle, \quad \tilde{\mu}^{A B} \equiv l^{-2}\left\langle\mu h^{A} h^{B}\right\rangle, \quad \bar{\mu}^{A B} \equiv l^{-4}\left\langle\mu g^{A} g^{B}\right\rangle, \\
& \tilde{P}^{A \beta} \equiv l^{-1}\left\langle\tilde{f}^{\beta} h^{A}\right\rangle, \quad \tilde{P}^{A} \equiv l^{-2}\left\langle\tilde{f} g^{A}\right\rangle,
\end{aligned}
$$

this model is represented by 
(i) the constitutive equations:

$$
\begin{aligned}
& N^{\alpha \beta}=\tilde{D}^{\alpha \beta \gamma \delta}\left(U_{\gamma, \delta}-b_{\gamma \delta} W\right)+D^{B \alpha \beta \gamma} Q_{\gamma}^{B}+\underline{\bar{D}^{B \alpha \beta \gamma}} Q_{\gamma, 1}^{B}-\underline{l^{2} L^{B \alpha \beta}} V^{B}, \\
& M^{\alpha \beta}=\widetilde{B}^{\alpha \beta \gamma \delta} W_{, \gamma \delta}+K^{B \alpha \beta} V^{B}+\underline{2 l \bar{K}^{B \alpha \beta}} V_{, 1}^{B}+\underline{l^{2} \breve{K}^{B \alpha \beta}} V_{, 11}^{B} \text {, } \\
& H^{A \beta}=D^{A \beta \gamma \delta}\left(U_{\gamma, \delta}-b_{\gamma \delta} W\right)+C^{A B \beta \gamma} Q_{\gamma}^{B}+\underline{\underline{l C}}{ }^{A B \beta \gamma} Q_{\gamma, 1}^{B}-\underline{l^{2} F^{A B \beta}} V^{B}, \\
& \bar{H}^{A \beta} \equiv \underline{l \bar{D}^{A \beta \gamma \delta}}\left(U_{\gamma, \delta}-b_{\gamma \delta} W\right)+\underline{l \bar{C}^{A B \beta \gamma}} Q_{\gamma}^{B}+\underline{l^{2} \tilde{C}^{A B \beta \gamma}} Q_{\gamma, 1}^{B}-\underline{l^{3} \bar{F}^{A B \beta}} V^{B} \text {, } \\
& G^{A} \equiv-\underline{l^{2} L^{A \gamma \delta}}\left(U_{\gamma, \delta}-b_{\gamma \delta} W\right)+K^{A \alpha \beta} W_{, \alpha \beta}-\underline{l^{2} F^{A B \gamma}} Q_{\gamma}^{B}-\underline{l^{3} \bar{F}^{A B \gamma}} Q_{\gamma, 1}^{B} \\
& +\left(S^{A B}+\underline{l^{4} \bar{L}^{A B}}\right) V^{B}+2 \underline{\breve{l R}^{A B}} V_{, 1}^{B}+\underline{l^{2} \widetilde{R}^{A B}} V_{, 11}^{B}, \\
& \widetilde{G}^{A}=\underline{l^{2} \breve{K}^{A \alpha \beta}} W_{, \alpha \beta}+\underline{l^{2} \widetilde{R}^{A B}} V^{B}+2 \underline{l^{3} \bar{R}^{A B}} V_{, 1}^{B}+\underline{l^{4} \stackrel{-A B}{R} V_{, 11}^{B},} \\
& \bar{G}^{A}=\underline{l \bar{K}^{A \alpha \beta}} W_{, \alpha \beta}+\underline{l \underline{R}} V^{B B}+2 \underline{l^{2} \tilde{S}^{A B}} V_{, 1}^{B}+\underline{l^{3} \bar{R}^{A B}} V_{, 11}^{B},
\end{aligned}
$$

(ii) the system of three averaged partial differential equations of motion for macrodisplacements $U_{\alpha}(\Theta, t), W(\Theta, t)$ :

$$
N_{, \alpha}^{\alpha \beta}-\tilde{\mu} a^{\alpha \beta} \ddot{U}_{\alpha}+f_{0}^{\beta}=0, \quad M_{, \alpha \beta}^{\alpha \beta}-b_{\alpha \beta} N^{\alpha \beta}+\bar{N}^{\alpha \beta} W_{, \alpha \beta}+\tilde{\mu} \ddot{W}-f_{0}=0,
$$

(iii) the system of $3 N$ partial differential equations for the fluctuation variables $Q_{\alpha}^{B}(\Theta, t)$, $V^{B}(\boldsymbol{\Theta}, t), B=1,2, \ldots, N$ :

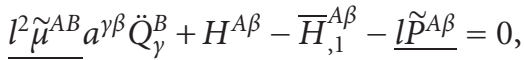

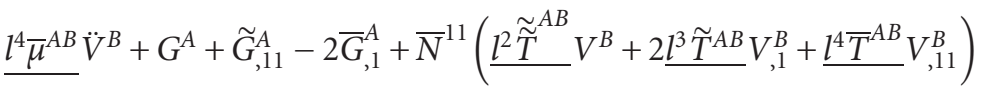

$$
\begin{aligned}
& +2 \bar{N}^{12}\left(\underline{l^{3} T} \breve{-A B}^{A B}+\underline{l^{2} \underline{T}^{A B}} V^{B}\right)-\bar{N}^{22} \underline{l^{2} T^{A B}} V^{B}-\underline{l^{2} \widetilde{P}^{A}}=0, \quad A, B=1,2, \ldots, N,
\end{aligned}
$$

where some terms depend explicitly on the period length $l$.

The above model has a physical sense provided that the basic unknowns $U_{\alpha}(\boldsymbol{\Theta}, t)$, $W(\Theta, t), Q_{\gamma}^{A}(\Theta, t), V^{A}(\Theta, t) \in \mathrm{SV}_{\Lambda}(T), A=1,2, \ldots, N$, that is, they are $\Lambda$-slowly varying functions of $\Theta^{2}$-midsurface parameter.

It can be observed that in the tolerance models (4.8), (4.9) we deal with $\bar{N}^{\alpha \beta}(t)>0$ if $\bar{N}^{\alpha \beta}(t)$ are compressive forces.

Taking into account (4.1) and (4.4) the shell displacement fields can be approximated by means of formulae

$$
\begin{gathered}
u_{\alpha}(\boldsymbol{\Theta}, t) \simeq U_{\alpha}(\boldsymbol{\Theta}, t)+h^{A}\left(\Theta^{1}, \Psi^{2}\right) Q_{\alpha}^{A}(\boldsymbol{\Theta}, t), \\
w(\Theta, t) \simeq W(\boldsymbol{\Theta}, t)+g^{A}\left(\Theta^{1}, \Psi^{2}\right) V^{A}(\boldsymbol{\Theta}, t), \quad A=1,2, \ldots, N,
\end{gathered}
$$


where the approximation $\simeq$ depends on the number of terms $h^{A}\left(\Theta^{1}, \Psi^{2}\right) Q_{\alpha}^{A}(\Theta, t), g^{A}\left(\Theta^{1}\right.$, $\left.\Psi^{2}\right) V^{A}(\boldsymbol{\Theta}, t)$.

The characteristic features of the derived models are the following.

(i) The model takes into account the effect of the cell size on the overall shell dynamics and stability; this effect is described by coefficients dependent on the period length $l$.

(ii) The model equations involve averaged coefficients which are independent of $\Theta^{2}$ midsurface parameter (i.e., they are constant in direction of periodicity) and are slowly varying functions of $\Theta^{1}$.

(iii) The number and form of boundary conditions for the macrodisplacements $U_{\alpha}(\Theta, t), W(\Theta, t)$ are the same as in the classical shell theory governed by (2.2)(2.4). The boundary conditions for the fluctuation variables $Q_{\gamma}^{A}(\Theta, t), V^{A}(\Theta, t)$ should be defined only on the boundaries $\Theta^{1}=$ const.

(iv) It is easy to see that in order to derive the governing equations (4.7)-(4.9), we have to postulate a priori periodic-shape (mode-shape) functions $h^{A}\left(\Theta^{1}, \Psi^{2}\right)$, $g^{A}\left(\Theta^{1}, \Psi^{2}\right), A=1,2, \ldots, N$, which can be derived from the periodic finite element method discretization of the cell or obtained as solutions to the periodic eigenvalue problem describing free vibrations of the cell, given by (4.5). Moreover, for uniperiodic shells the shape (mode-shape) functions are periodic in only one direction; in this work they are $l$-periodic functions only of the $\Theta^{2}$-midsurface parameter.

Assuming that the cylindrical shell under consideration has material and geometrical properties independent of $\Theta^{1}$ we obtain governing equations (4.7)-(4.9) with constant averaged coefficients. Moreover, in this case the mode-shape functions $h^{A}, g^{A}, A=$ $1,2, \ldots, N$, are also independent of $\Theta^{1}$-midsurface parameter.

For a homogeneous shell $\mu(\boldsymbol{\Theta}), D^{\alpha \beta \gamma \delta}(\boldsymbol{\Theta})$ and $B^{\alpha \beta \gamma \delta}(\boldsymbol{\Theta}), \boldsymbol{\Theta} \in \Omega$, are constant and because $\left\langle\mu h^{A}\right\rangle=\left\langle\mu g^{A}\right\rangle=0$ we obtain $\left\langle h^{A}\right\rangle=\left\langle g^{A}\right\rangle=0$, and hence $\left\langle h_{, \alpha}^{A}\right\rangle=\left\langle g_{, \alpha}^{A}\right\rangle=\left\langle g_{, \alpha \beta}^{A}\right\rangle=0$. In this case (4.8) reduce to the well-known linear-elastic shell equations of motion for macrodisplacements $U_{\alpha}(\boldsymbol{\Theta}, t), W(\boldsymbol{\Theta}, t)$, and independently for $Q_{\alpha}^{A}(\boldsymbol{\Theta}, t), V^{A}(\boldsymbol{\Theta}, t)$ we arrive at a system of $N$ differential equations. In the case under the condition $\tilde{f}^{\beta}=\tilde{f}=0$ and for initial conditions $Q_{\alpha}^{A}\left(\boldsymbol{\Theta}, t_{0}\right)=V^{A}\left(\Theta, t_{0}\right)=0, A=1,2, \ldots, N$, we obtain $Q_{\alpha}^{A}=V^{A}=0$; hence the constitutive equations (4.7) and equations of motion (4.8) reduce to the starting equations (2.3) and (2.4), respectively.

In the next section the homogenized model of uniperiodic cylindrical shells under consideration will be derived as a special case of (4.7)-(4.9).

\section{Governing equations of the asymptotic model}

The simplified model of uniperiodic cylindrical shells, called homogenized or asymptotic, can be derived directly from the tolerance model (4.7)-(4.9) by a limit passage $l \rightarrow 0$, that is, by neglecting the terms which depend on the period length $l$. Hence, (4.9) yield

$$
C^{A B \beta \gamma} Q_{\gamma}^{B}=-D^{A \beta \gamma \delta}\left(U_{\gamma, \delta}-b_{\gamma \delta} W\right), \quad S^{A B} V^{A}=-K^{B \gamma \delta} W_{, \gamma \delta}
$$


From the positive definiteness of the strain energy it follows that $N \times N$ matrix of elements $S^{A B}$ is nonsingular, and the linear transformation determined by components $C^{A B \beta \gamma}$ is invertible. Hence a solution to (5.1) can be written in the form

$$
Q_{\gamma}^{B}=-G_{\gamma \eta}^{B C} D^{C \eta \mu \vartheta}\left(U_{\mu, \vartheta}-b_{\mu \vartheta} W\right), \quad V^{A}=-E^{A B} K^{B \gamma \delta} W_{, \gamma \delta}
$$

where $G_{\alpha \beta}^{A B}$ and $E^{A B}$ are defined by $G_{\alpha \beta}^{A B} C^{B C \beta \gamma}=\delta_{\alpha}^{\gamma} \delta^{A C}, E^{A B} S^{B C}=\delta^{A C}$.

Setting

$$
D_{\text {eff }}^{\alpha \beta \gamma \delta} \equiv \widetilde{D}^{\alpha \beta \gamma \delta}-D^{A \alpha \beta \eta} G_{\eta \xi}^{A B} D^{B \xi \gamma \delta}, \quad B_{\text {eff }}^{\alpha \beta \gamma \delta} \equiv \widetilde{B}^{\alpha \beta \gamma \delta}-K^{A \alpha \beta} E^{A B} K^{B \gamma \delta}
$$

and substituting the expression (5.2) into the constitutive equations $(4.7)_{1,2}$, in which the underlined terms are neglected, we arrive at the homogenized (asymptotic) shell model governed by

(i) equations of motion:

$$
\begin{gathered}
D_{\mathrm{eff}}^{\alpha \beta \gamma \delta}\left(U_{\gamma, \delta \alpha}-b_{\gamma \delta} W_{, \alpha}\right)-\langle\mu\rangle a^{\alpha \beta} \ddot{U}_{\alpha}+f_{0}^{\beta}=0, \\
B_{\mathrm{eff}}^{\alpha \beta \gamma \delta} W_{, \alpha \beta \gamma \delta}-b_{\alpha \beta} D_{\mathrm{eff}}^{\alpha \beta \gamma \delta}\left(U_{\gamma, \delta}-b_{\gamma \delta} W\right)+\bar{N}^{\alpha \beta} W_{, \alpha \beta}+\langle\mu\rangle \ddot{W}-f_{0}=0,
\end{gathered}
$$

(ii) constitutive equations:

$$
N^{\alpha \beta}=D_{\mathrm{eff}}^{\alpha \beta \gamma \delta}\left(U_{\gamma, \delta}-b_{\gamma \delta} W\right), \quad M^{\alpha \beta}=-B_{\mathrm{eff}}^{\alpha \beta \gamma \delta} W_{, \gamma \delta},
$$

where $D_{\text {eff }}^{\alpha \beta \gamma \delta}, B_{\text {eff }}^{\alpha \beta \gamma \delta}$ are called the effective stiffnesses.

The above obtained homogenized model governed by (5.4), (5.5) is not able to describe the length-scale effect on the overall shell behavior being independent of the period length $l$.

In order to show differences between the results obtained from the tolerance uniperiodic shell model (4.7)-(4.9) and from the homogenized model (5.4), (5.5), free vibrations of a special case of uniperiodic cylindrical shell will be analyzed in the next section.

\section{Applications}

The objective of this section is to determine and investigate free vibrations of an open circular cylindrical shell with $L_{1}, L_{2}$ as its axial length and arc length along the lines of principal curvature of the shell midsurface, respectively, and with $\delta, R$ as its constant thickness and its midsurface curvature radius, respectively. The shell is reinforced by two families of densely spaced ribs, which are parallel to the generatrix of cylindrical surface and are periodically distributed along the lines of the shell midsurface principal curvature (cf. Figure 6.1). The stiffeners of both kinds are assumed to have constant rectangular crosssections with $A_{1}, A_{2}$ as their areas and with $I_{1}, I_{2}$ as their moments of inertia. Moreover, the gravity centers of the stiffener cross-sections are situated on the shell midsurface.

It is assumed that both the shell and stiffeners are made of homogeneous isotropic materials; and let us denote by $E, v$ Young's modulus and Poisson's ratio of the shell material, respectively, and by $E_{1}, E_{2}$ Young's moduli of the rib materials. At the same time $\mu_{0}$ 


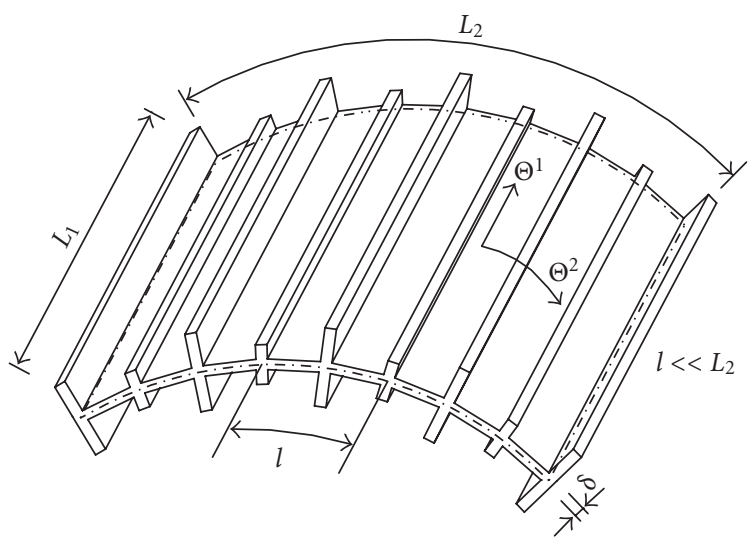

Figure 6.1. A shell with two families of uniperiodically spaced ribs.

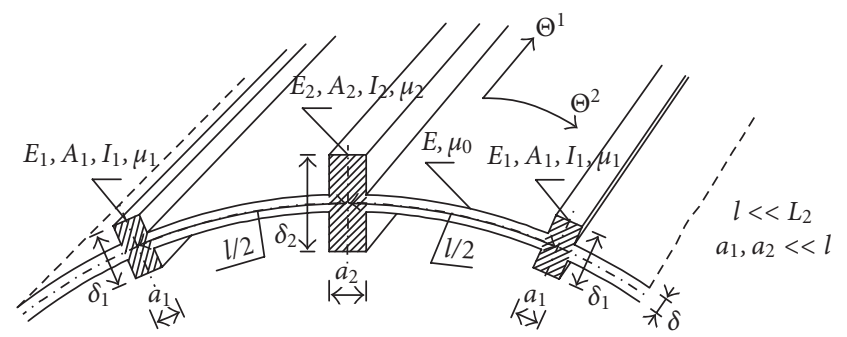

Figure 6.2. A fragment of the stiffened shell cross-section.

stands for the constant shell mass density per midsurface unit area and $\mu_{1}, \mu_{2}$ stand for the constant mass densities of the stiffeners per the stiffener unit length (cf. Figure 6.2).

Let $\Theta^{1}, \Theta^{2}$ be axial and arc coordinates on the shell midsurface $\mathcal{M}$, respectively, and let $\Theta^{2}$-coordinate lines coincide with the lines of principal curvature of this surface.

It is assumed that the edges of the shell lie on the coordinate lines $\Theta^{1}=0, \Theta^{1}=L_{1}$ and $\Theta^{2}=0, \Theta^{2}=L_{2}$ and that all four edges are simply supported.

In agreement with considerations in Section 2, on $O \Theta^{1} \Theta^{2}$-plane we define $l$ as the period of the stiffened shell structure in $\Theta^{2}$-direction, which represent the distance (i.e., the arc length measured along the lines of midsurface principal curvature) between axes of two neighboring ribs belonging to the same family (cf. Figures 6.1 and 6.2). It means that the axes of undeformed stiffeners are situated on the lines $\Theta^{2}=n_{1} l, n_{1}=0,1,2, \ldots, M$, and $\Theta^{2}=n_{2} l+l / 2, n_{2}=0,1,2, \ldots,(M-1), L_{2}=(M-1) l$, where $(2 M-1)$ is the number of stiffeners (cf. Figure 6.1).

The period $l$ has to satisfy the conditions $\delta \ll l \ll L_{2}$. It means that the number of stiffeners has to be very large. We also assume that $L_{1} \geq L_{2}$; it follows that $l$ satisfies the condition $l \ll L_{1}$. 


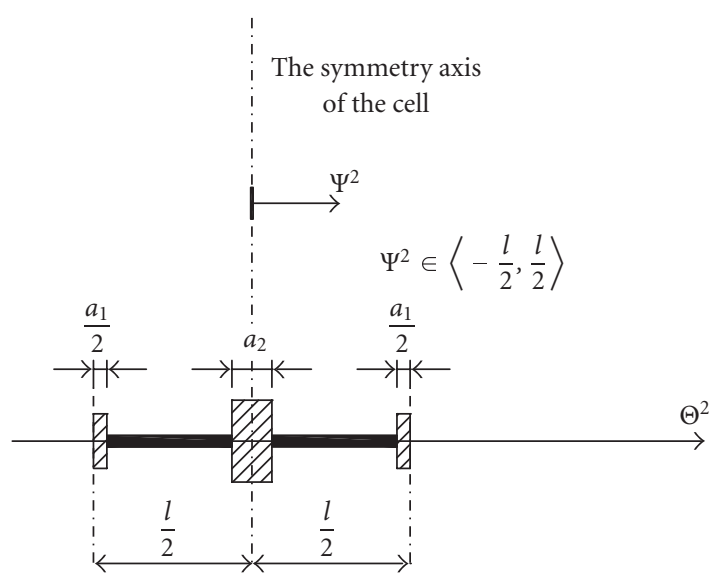

Figure 6.3. A periodicity cell along the $O \Theta^{2}$-axis direction on $O \Theta^{1} \Theta^{2}$-plane, $a_{1}, a_{2} \ll l$.

Denoting by $a_{1}, a_{2}$ the widths of the ribs (cf. Figure 6.2) we assume that $a_{1}, a_{2} \ll l$ and hence the torsional rigidity of stiffeners can be neglected.

The tensile and bending rigidities of the stiffeners are constant. The rigidities of the shell are also constant and described by the components of the shell stiffness tensors $D_{0}^{\alpha \beta \gamma \delta}, B_{0}^{\alpha \beta \gamma \delta}$ given by

$$
D_{0}^{\alpha \beta \gamma \delta}=D H^{\alpha \beta \gamma \delta}, \quad B_{0}^{\alpha \beta \gamma \delta}=B H^{\alpha \beta \gamma \delta},
$$

where

$$
\begin{gathered}
D=\frac{E \delta}{\left(1-\nu^{2}\right)}, \quad B=\frac{E \delta^{3}}{12\left(1-\nu^{2}\right)}, \\
H^{\alpha \beta \gamma \delta}=0.5\left[a^{\alpha \gamma} a^{\beta \delta}+a^{\alpha \delta} a^{\beta \gamma}+\nu\left(\in^{\alpha \gamma} \in^{\beta \delta}+\in^{\alpha \delta} \in \in^{\beta \gamma}\right)\right],
\end{gathered}
$$

with $a^{\alpha \gamma}, \in^{\alpha \gamma}$ as contravariant first midsurface tensor and Ricci bivector, respectively. After some manipulations we obtain the following expressions for the nonzero components of tensor $H^{\alpha \beta \gamma \delta}$ :

$$
\begin{gathered}
H^{1111}=H^{2222}=1, \quad H^{1122}=H^{2211}=v, \\
H^{1212}=H^{1221}=H^{2121}=H^{2112}=\frac{1-\nu}{2} .
\end{gathered}
$$

We define the periodicity cell $\Lambda$ on $O \Theta^{1} \Theta^{2}$-plane by means of $\Lambda \equiv(-l / 2, l / 2), \Lambda\left(\Theta^{1}\right.$, $\left.\Theta^{2}\right) \equiv\left(\Theta^{1}, \Theta^{2}-l / 2, \Theta^{1}, \Theta^{2}+l / 2\right),\left(\Theta^{1}, \Theta^{2}\right) \in \Omega_{\Lambda}, \Omega_{\Lambda}:=\{\Theta \in \Omega, \Lambda(\Theta) \in \Omega\}$. The cell $\Lambda$ is shown in Figure 6.3. Setting $\Psi^{2} \in\langle-l / 2, l / 2\rangle$, we assume that the cell $\Lambda$ has a symmetry axis for $\Psi^{2}=0$.

The periodically ribbed shell under consideration will be treated as a nonstiffened shell with constant thickness $\delta$, made of a certain nonhomogeneous material. Let us denote by 
$D^{\alpha \beta \gamma \delta}, B^{\alpha \beta \gamma \delta}$, and $\mu$ the stiffness tensors and mass density of this nonribbed shell, respectively. The shell's tensile $D^{1111}$ and bending $B^{1111}$ stiffnesses in the axial direction are l-periodic function in $\Theta^{2}$, being independent of $\Theta^{1}$, and are different from tensile $D^{2222}$ and bending $B^{2222}$ rigidities in circumferential direction, being constant functions. The shell's mass density $\mu$ is $l$-periodic function in $\Theta^{2}$, being independent of $\Theta^{1}$.

Under assumption that the torsional rigidity of stiffeners is neglected, the components of the shell stiffness tensors $D^{\alpha \beta \gamma \delta}, B^{\alpha \beta \gamma \delta}$, except for $D^{1111}, B^{1111}$, are constant and given by $D^{\alpha \beta \gamma \delta}=D_{0}^{\alpha \beta \gamma \delta}, B^{\alpha \beta \gamma \delta}=B_{0}^{\alpha \beta \gamma \delta}$. Inside the cell $\Lambda$, the tensile rigidity $D^{1111}\left(\Psi^{2}\right)$ and bending rigidity $B^{1111}\left(\Psi^{2}\right)$ take the following form:

$$
\begin{gathered}
D^{1111}\left(\Psi^{2}\right)= \begin{cases}D_{0}^{1111}=D & \text { for } \Psi^{2} \in\left(-\frac{l}{2}, \frac{l}{2}\right)-\{0\}, \\
\frac{E_{1} A_{1}}{2} & \text { for } \Psi^{2}=-\frac{l}{2} \text { and } \Psi^{2}=\frac{l}{2}, \\
E_{2} A_{2} & \text { for } \Psi^{2}=0,\end{cases} \\
B^{1111}\left(\Psi^{2}\right)= \begin{cases}B_{0}^{1111}=B & \text { for } \Psi^{2} \in\left(-\frac{l}{2}, \frac{l}{2}\right)-\{0\}, \\
\frac{E_{1} I_{1}}{2} & \text { for } \Psi^{2}=-\frac{l}{2} \text { and } \Psi^{2}=\frac{l}{2}, \\
E_{2} I_{2} & \text { for } \Psi^{2}=0 .\end{cases}
\end{gathered}
$$

Inside the cell $\Lambda$, the shell mass density is given by

$$
\mu\left(\Psi^{2}\right)= \begin{cases}\mu_{0} & \text { for } \Psi^{2} \in\left(-\frac{l}{2}, \frac{l}{2}\right)-\{0\} \\ \mu_{1} & \text { for } \Psi^{2}=-\frac{l}{2} \text { and } \Psi^{2}=\frac{l}{2} \\ \mu_{2} & \text { for } \Psi^{2}=0 .\end{cases}
$$

Taking into account definition (2.1) we obtain for functions $D^{1111}\left(\Psi^{2}\right), B^{1111}\left(\Psi^{2}\right)$, $\mu\left(\Psi^{2}\right)$ given above the following averaged values:

$$
\begin{aligned}
\widetilde{D}^{1111} & \equiv\left\langle D^{1111}\right\rangle=\frac{D+\left(E_{1} A_{1}+E_{2} A_{2}\right)}{l}, \\
\widetilde{B}^{1111} & \equiv\left\langle B^{1111}\right\rangle=\frac{B+\left(E_{1} I_{1}+E_{2} I_{2}\right)}{l}, \\
\tilde{\mu} & \equiv\langle\mu\rangle=\mu_{0}+\frac{\left(\mu_{1}+\mu_{2}\right)}{l} .
\end{aligned}
$$

In order to investigate free vibrations, we assume that the external forces $f^{\beta}, f$ are equal to zero. We also assume that $\bar{N}^{\alpha \beta}(t)=0$. 
Considerations will be restricted to the transverse vibrations of the shell; it means that the forces of inertia in directions tangential to the shell midsurface will be neglected.

For the sake of simplicity, we restrict our considerations to the first terms in series $h^{A}(\cdot) Q_{\alpha}^{A}(\cdot, t), g^{A}(\cdot) V^{A}(\cdot, t), A=1,2, \ldots, N$, that is, $A=N=1$. Hence, we introduce only two $l$-periodic mode-shape functions $h\left(\Psi^{2}\right) \equiv h^{1}\left(\Psi^{2}\right), g\left(\Psi^{2}\right) \equiv g^{1}\left(\Psi^{2}\right), \Psi^{2} \in\langle-l / 2, l / 2\rangle$, which have to satisfy condition $\langle\mu h\rangle=\langle\mu g\rangle=0$ and the values of which are of order $O(l)$ and $O\left(l^{2}\right)$, respectively. Functions $h\left(\Psi^{2}\right), g\left(\Psi^{2}\right)$ can be obtained as solutions to periodic eigenvalue problem on the cell given by (4.5) and hence they are referred to the lowest natural vibration modes in directions tangent and normal to the shell midsurface, respectively.

Taking into account the symmetric form of the cell (cf. Figure 6.3) we assume that the shape function $h\left(\Psi^{2}\right)$ is antisymmetric on the cell $\Lambda$ while the shape function $g\left(\Psi^{2}\right)$ is symmetric.

Taking into account the fact that, except for $D^{1111}, B^{1111}$, the components of the shell stiffness tensors $D^{\alpha \beta \gamma \delta}, B^{\alpha \beta \gamma \delta}$ are constant and that the functions $h\left(\Psi^{2}\right), g\left(\Psi^{2}\right)$ are independent of $\Theta^{1}$ as well as bearing in mind the symmetric form of the cell and the symmetric form of function $g\left(\Psi^{2}\right)$ as well as antisymmetric form of function $h\left(\Psi^{2}\right)$, it can be shown that only the following averages in (4.6) are different from zero: $\widetilde{D}^{\alpha \beta \gamma \delta}, \widetilde{B}^{\alpha \beta \gamma \delta}, L^{A 11}$, $L^{A 22}, \breve{K}^{A 11}, \breve{K}^{A 22}, C^{A B 11}, C^{A B 22}, \widetilde{C}^{A B 11}, \widetilde{C}^{A B 22}, F^{A B 2}, S^{A B}, \bar{L}^{A B}, \widetilde{R}^{A B}, \overbrace{R}^{A B}, \widetilde{S}^{A B}, A, B=1$. Under assumption $A=B=N=1$ we introduce the following denotations for these nonzero averages:

$$
\begin{gathered}
L^{11} \equiv L^{A 11}, \quad L^{22} \equiv L^{A 22}, \quad \breve{K}^{11} \equiv \breve{K}^{A 11}, \quad \breve{K}^{22} \equiv \breve{K}^{A 22}, \\
C^{11} \equiv C^{A B 11}, \quad C^{22} \equiv C^{A B 22}, \quad \widetilde{C}^{11} \equiv \widetilde{C}^{A B 11}, \quad \widetilde{C}^{22} \equiv \widetilde{C}^{A B 22}, \quad F^{2} \equiv F^{A B 2}, \\
S \equiv S^{A B}, \quad \bar{L} \equiv \bar{L}^{A B}, \quad \widetilde{R} \equiv \widetilde{R}^{A B}, \quad \widetilde{R} \equiv \widetilde{R}^{A B}, \quad \widetilde{S} \equiv \widetilde{S}^{A B}, \quad A, B=1 .
\end{gathered}
$$

We also denote $Q_{1}(\boldsymbol{\Theta}) \equiv Q_{1}^{1}(\boldsymbol{\Theta}), Q_{2}(\boldsymbol{\Theta}) \equiv Q_{2}^{1}(\boldsymbol{\Theta}), V(\boldsymbol{\Theta}) \equiv V^{1}(\boldsymbol{\Theta}), \boldsymbol{\Theta} \equiv\left(\Theta^{1}, \Theta^{2}\right)$.

Bearing in mind the conditions and denotations given above we will derive below the formulae for free vibration frequencies of the considered uniperiodic shell by using both the tolerance model given by (4.7)-(4.9) and the homogenized model presented by (5.4), (5.5).

6.1. The tolerance model. Now, the governing equations (4.8), (4.9) of the tolerance model are separated into independent equation for $Q_{1}(\Theta, t): C^{11} Q_{1}-l^{2} \widetilde{C}^{11} Q_{1,11}=0$, which yields $Q_{1}=0$, and the system of five equations for macrodisplacements $U_{1}(\boldsymbol{\Theta}, t)$, $U_{2}(\boldsymbol{\Theta}, t), W(\boldsymbol{\Theta}, t)$ and fluctuation variables $Q_{2}(\boldsymbol{\Theta}, t), V(\boldsymbol{\Theta}, t), \boldsymbol{\Theta} \equiv\left(\Theta^{1}, \Theta^{2}\right)$, being $\Lambda$ slowly-varying functions in $\Theta^{2}$

$$
\begin{gathered}
\widetilde{D}^{1111} U_{1,11}+D\left[(1-v) 2^{-1} U_{1,22}+(1+\nu) 2^{-1} U_{2,12}+\nu R^{-1} W_{, 1}\right]-l^{2} L^{11} V_{, 1}=0, \\
D\left[(1+\nu) 2^{-1} U_{1,12}+(1-v) 2^{-1} U_{2,11}+U_{2,22}+R^{-1} W_{, 2}\right]-l^{2} L^{22} V_{, 2}=0,
\end{gathered}
$$




$$
\begin{gathered}
D R^{-1}\left(\nu U_{1,1}+U_{2,2}+R^{-1} W\right)+\widetilde{B}^{1111} W_{, 1111}+B\left(2 W_{, 1122}+W_{, 2222}\right)+\tilde{\mu} \ddot{W} \\
+l^{2} \breve{K}^{11} V_{, 1111}+l^{2} \breve{K}^{22} V_{, 1122}-R^{-1} l^{2} L^{22} V=0, \\
C^{22} Q_{2}-l^{2} \widetilde{C}^{22} Q_{2,11}-l^{2} F^{2} V=0, \\
-l^{2} L^{11} U_{1,1}-l^{2} L^{22}\left(U_{2,2}+R^{-1} W\right)+l^{2} \breve{K}^{11} W_{, 1111}+l^{2} \widetilde{K}^{-22} W_{, 2211} \\
-l^{2} F^{2} Q_{2}+\left(S+l^{4} \bar{L}\right) V+2 l^{2}(\widetilde{R}-2 \widetilde{S}) V_{, 11}+l^{4} \widetilde{R} V_{, 1111}+l^{4} \bar{\mu} \ddot{V}=0,
\end{gathered}
$$

where some terms depend explicitly on the period length $l$; the averages $\widetilde{D}^{1111}, \widetilde{B}^{1111}, \tilde{\mu}$ are defined by (6.6) and the remaining ones are given by (6.7) and (4.6).

It is easy to see that all coefficients of the above equations are constant.

Solutions to (6.1) satisfying boundary conditions for a simply supported shell can be assumed in the form (see [1])

$$
\begin{gathered}
U_{1}=\tilde{U}_{1} \cos \left(\alpha \Theta^{1}\right) \sin \left(\beta \Theta^{2}\right) \cos (\omega t), \quad U_{2}=\tilde{U}_{2} \sin \left(\alpha \Theta^{1}\right) \cos \left(\beta \Theta^{2}\right) \cos (\omega t), \\
Q_{2}=\widetilde{Q}_{2} \sin \left(\alpha \Theta^{1}\right) \sin \left(\beta \Theta^{2}\right) \cos (\omega t), \quad W=\widetilde{W} \sin \left(\alpha \Theta^{1}\right) \sin \left(\beta \Theta^{2}\right) \cos (\omega t), \\
V=\tilde{V} \sin \left(\alpha \Theta^{1}\right) \sin \left(\beta \Theta^{2}\right) \cos (\omega t),
\end{gathered}
$$

where $\alpha=\pi / L_{1}, \beta=\pi / L_{2}$.

Substituting the right-hand sides of (6.9) into (6.1) we obtain the system of five linear homogeneous algebraic equations for $\widetilde{U}_{1}, \widetilde{U}_{2}, \widetilde{Q}_{2}, \widetilde{W}, \widetilde{V}$. For a nontrivial solution the determinant of the coefficients of these equations must equal zero. In this manner we arrive at the characteristic equation for the lowest frequency $\omega$ of the transverse free vibrations of the shell. Setting $\omega^{t m} \equiv \omega$ and introducing the following notations:

$$
\begin{gathered}
\tilde{a}_{1} \equiv \alpha^{2} \tilde{D}^{1111}+\beta^{2} D(1-v) 2^{-1}, \quad \tilde{a}_{2} \equiv \alpha \beta D(1+\nu) 2^{-1}, \\
\tilde{a}_{3} \equiv-\alpha D \nu R^{-1}, \quad \tilde{a}_{4} \equiv D\left[\alpha^{2}(1-\nu) 2^{-1}+\beta^{2}\right], \quad \tilde{a}_{5} \equiv-\beta D R^{-1}, \\
\tilde{a}_{6} \equiv \alpha^{4} \tilde{B}^{1111}+B\left(2 \alpha^{2} \beta^{2}+\beta^{4}\right)+D R^{-2}, \\
\tilde{a} \equiv-\left(\tilde{a}_{2}\right)^{2}\left(\tilde{a}_{1}\right)^{-1}+\tilde{a}_{4}, \quad \tilde{b} \equiv-\tilde{a}_{2} \tilde{a}_{3}\left(\tilde{a}_{1}\right)^{-1}+\tilde{a}_{5}, \quad \tilde{c} \equiv-\tilde{a}_{2}\left(\tilde{a}_{1}\right)^{-1} \alpha L^{11}+\beta L^{22}, \\
\tilde{d} \equiv-\left(\tilde{a}_{3}\right)^{2}\left(\tilde{a}_{1}\right)^{-1}+\tilde{a}_{6}, \quad \tilde{e} \equiv-\tilde{a}_{3}\left(\tilde{a}_{1}\right)^{-1} \alpha L^{11}-R^{-1} L^{22}+\alpha^{2} \beta^{2} \bar{K}^{22}, \\
\tilde{f} \equiv-\alpha^{2}\left(\tilde{a}_{1}\right)^{-1}\left(L^{11}\right)^{2}-\left(F^{2}\right)^{2}\left(C^{22}\right)^{-1}\left[1+\alpha^{2} l^{2} \tilde{C}^{22}\left(C^{22}\right)^{-1}\right]^{-1}+\bar{L} \\
+l^{-4} S\left[1+2 \alpha^{2} l^{2}(2 \tilde{S}-\tilde{R}) S^{-1}+\alpha^{4} l^{4} \tilde{R} S^{-1}\right],
\end{gathered}
$$


this equation has the following form:

$$
\left(\omega^{t m}\right)^{4}-\left(\omega^{t m}\right)^{2}\left[\frac{1}{\bar{\mu}}\left(\tilde{f}-\frac{\tilde{c}^{2}}{\tilde{a}}\right)+\frac{1}{\tilde{\mu}}\left(\tilde{d}-\frac{\tilde{b}^{2}}{\tilde{a}}\right)\right]+\frac{1}{\tilde{\mu} \bar{\mu}}\left[\tilde{f}\left(\tilde{d}-\frac{\tilde{b}^{2}}{\tilde{a}}\right)+\frac{\tilde{c}}{\tilde{a}}(2 \tilde{b} \tilde{e}-\tilde{d} \tilde{c})-\tilde{e}^{2}\right]=0 .
$$

In the above equation the period length $l$ is comprised in the term $\tilde{f}$.

Because the shell under consideration satisfies the condition $l / L_{1} \ll 1$, that is, $\alpha l \ll 1$, in the sequel the simplified form of (6.12) will be applied, in which the terms

$$
(\alpha l)^{2} \widetilde{C}^{22}\left(C^{22}\right)^{-1}, \quad 2(\alpha l)^{2}(2 \widetilde{S}-\widetilde{R}) S^{-1}, \quad(\alpha l)^{4} \widehat{R} \widetilde{S}^{-1}
$$

can be neglected as small in comparison with unity. Hence, after setting

$$
\tilde{f}^{\prime} \equiv-\alpha^{2}\left(\tilde{a}_{1}\right)^{-1}\left(L^{11}\right)^{2}-\left(F^{2}\right)^{2}\left(C^{22}\right)^{-1}+\bar{L},
$$

the term $\tilde{f}$ in $(6.12)$, which is defined by $(6.11)_{6}$, simplifies to

$$
\tilde{f} \approx \tilde{f}^{\prime}+l^{-4} S .
$$

Taking into account (6.14) and (6.15) and using the notations

$$
\eta \equiv \tilde{f}^{\prime}-\frac{\tilde{c}^{2}}{\tilde{a}}, \quad \tilde{\eta} \equiv \tilde{d}-\frac{\tilde{b}^{2}}{\tilde{a}}, \quad \xi \equiv \tilde{f}^{\prime}\left(\tilde{d}-\frac{\tilde{b}^{2}}{\tilde{a}}\right)+\frac{\tilde{c}}{\tilde{a}}(2 \tilde{b} \tilde{e}-\tilde{d} \tilde{c})-\tilde{e}^{2},
$$

we obtain from (6.12) the following formulae for fundamental lower free vibration frequency $\left(\omega_{-}^{t m}\right)^{2}$ and for the additional higher free vibration frequency $\left(\omega_{+}^{t m}\right)^{2}$, caused by the uniperiodic structure of the shell under consideration

$$
\begin{aligned}
& \left(\omega_{-}^{t m}\right)^{2}=\frac{1}{2}\left(\frac{S}{\bar{\mu} l^{4}}+\frac{\eta}{\bar{\mu}}+\frac{\tilde{\eta}}{\tilde{\mu}}\right)-\frac{1}{2} \sqrt{\left(\frac{S}{\bar{\mu} l^{4}}+\frac{\eta}{\bar{\mu}}+\frac{\tilde{\eta}}{\tilde{\mu}}\right)^{2}-\frac{4}{\tilde{\mu} \bar{\mu}}\left(\frac{S \tilde{\eta}}{l^{4}}+\xi\right),} \\
& \left(\omega_{+}^{t m}\right)^{2}=\frac{1}{2}\left(\frac{S}{\bar{\mu} l^{4}}+\frac{\eta}{\bar{\mu}}+\frac{\tilde{\eta}}{\tilde{\mu}}\right)+\frac{1}{2} \sqrt{\left(\frac{S}{\bar{\mu} l^{4}}+\frac{\eta}{\bar{\mu}}+\frac{\tilde{\eta}}{\tilde{\mu}}\right)^{2}-\frac{4}{\tilde{\mu} \bar{\mu}}\left(\frac{S \tilde{\eta}}{l^{4}}+\xi\right)} .
\end{aligned}
$$

The results depend on the period length $l$.

6.2. The homogenized model. In order to evaluate obtained results, let us consider the above problem within the homogenized (i.e., asymptotic) model. From (6.1), after neglecting the terms of orders $O\left(l^{2}\right)$ and $O\left(l^{4}\right)$, we obtain the following governing relations of the homogenized model:

$$
\begin{gathered}
\widetilde{D}^{1111} U_{1,11}+D\left[(1-\nu) 2^{-1} U_{1,22}+(1+\nu) 2^{-1} U_{2,12}+\nu R^{-1} W_{, 1}\right]=0, \\
D\left[(1+\nu) 2^{-1} U_{1,12}+(1-\nu) 2^{-1} U_{2,11}+U_{2,22}+R^{-1} W_{, 2}\right]=0, \\
D R^{-1}\left(\nu U_{1,1}+U_{2,2}+R^{-1} W\right)+\widetilde{B}^{1111} W_{, 1111}+B\left(2 W_{, 1122}+W_{, 2222}\right)+\tilde{\mu} \ddot{W}+=0 .
\end{gathered}
$$


The obtained above model is not able to describe the length-scale effect on the overall shell dynamics being independent of the period length $l$.

It is easy to see that there are no fluctuation variables in the asymptotic model (6.18) derived here. It means that $U_{1}=u_{1}, U_{2}=u_{2}, W=w$, and hence the governing equations (6.18) coincide with the well-known equations of dynamic problems for stringerstiffened cylindrical shells; see [1].

The solutions to (6.18) can be assumed in the form (6.9) $)_{1,2,4}$. Substituting the solutions to $(6.18)$ we obtain the system of three linear homogeneous algebraic equations in $\widetilde{U}_{1}, \widetilde{U}_{2}$, $\widetilde{W}$. For $\widetilde{U}_{1} \neq 0, \widetilde{U}_{2} \neq 0, \widetilde{W} \neq 0$ we arrive at the formula for the lowest frequency $\omega$ of the transverse free vibrations of the shell. Setting $\omega^{h m} \equiv \omega$, this formula has the form

$$
\left(\omega^{h m}\right)^{2}=\frac{\tilde{\eta}}{\widetilde{\mu}}
$$

where $\tilde{\mu}$ and $\tilde{\eta}$ are given by $(6.6)_{3}$ and $(6.16)_{2}$, respectively.

It is easy to see that in the above formula the cell size is neglected and that in the framework of the asymptotic model it is not possible to determine the additional higher free vibration frequency, caused by the periodic structure of the shell.

In the next subsection a comparison of the results obtained in Sections 6.1 and 6.2 will be presented.

6.3. A comparison of results. In order to compare the lower free vibration frequency given by $(6.17)_{1}$, which has been derived from the tolerance model with that given by (6.19) obtained from the homogenized model, let us denote $\varepsilon \equiv l^{4}$. Under this notation and after some manipulations, the first one from (6.17) takes the form

$$
\left(\omega_{-}^{t m}\right)^{2}=\frac{1}{2}\left(\frac{S}{\bar{\mu} \varepsilon}+\frac{\eta}{\bar{\mu}}+\frac{\tilde{\eta}}{\tilde{\mu}}\right)-\frac{S}{2 \bar{\mu} \varepsilon} \sqrt{1+\left(\frac{2 \eta}{S}-\frac{2 \bar{\mu} \tilde{\eta}}{\tilde{\mu} S}\right) \varepsilon+\left(\frac{\eta}{S}+\frac{\bar{\mu} \tilde{\eta}}{\tilde{\mu} S}-\frac{4 \bar{\mu}}{\tilde{\mu} S^{2}} \xi\right) \varepsilon^{2} .}
$$

Let us observe that the constant $\varepsilon$ can be treated as a small parameter. Representing the square root in the above formula for $\left(\omega_{-}^{t m}\right)^{2}$ in the form of the power series with respect to $\varepsilon$, we obtain

$$
\left(\omega_{-}^{t m}\right)^{2}=\frac{\tilde{\eta}}{\tilde{\mu}}+O(\varepsilon)
$$

Taking into account (6.19), we arrive finally at the interrelation

$$
\left(\omega_{-}^{t m}\right)^{2}=\left(\omega^{h m}\right)^{2}+O\left(l^{4}\right)
$$

between the values of squares of free vibration frequencies $\left(\omega_{-}^{t m}\right)^{2}$ and $\left(\omega^{h m}\right)^{2}$ obtained within frameworks of the tolerance and homogenized models, respectively. It means that the differences between lower value of the free vibration frequency derived from the tolerance model and free vibration frequency obtained from the asymptotic one are negligibly small. Thus, in this case, the effect of the period length $l$ on the free vibrations of the shell under consideration can be neglected and we can use the asymptotic model represented by (5.4), (5.5) instead of the nonasymptotic tolerance model given by (4.7)-(4.9). 
6.4. Conclusions. Summarizing the results obtained in this section it can be concluded that

(i) contrary to homogenized (asymptotic) model, the proposed non-asymptotic one describes the effect of the period length $l$ on the shell dynamics;

(ii) in the framework of the nonasymptotic tolerance model proposed in this contribution, the fundamental lower and additional higher free vibration frequencies can be derived. The higher free vibration frequency, caused by a periodic structure of the stiffened shell cannot be determined using the homogenized (i.e., asymptotic) model;

(iii) differences between lower values of the free vibration frequencies derived from the tolerance model and free vibration frequencies obtained from the asymptotic one are negligibly small; the squares of free vibration frequencies calculated from the asymptotic model are approximations of order $O\left(l^{4}\right)$ of the squares of lower free vibration frequencies derived from the tolerance model, that is, $\left(\omega_{-}^{t m}\right)^{2}=$ $\left(\omega^{h m}\right)^{2}+O\left(l^{4}\right)$. Thus the effect of the period length $l$ on the shell dynamics can be neglected and hence the homogenized model given by (5.4), (5.5) is sufficient from the point of view of calculation for the problem of determining the free vibration frequencies of uniperiodically densely stiffened cylindrical shells under consideration.

\section{Final remarks}

The subject matter of this contribution is a thin linear-elastic cylindrical shell having a periodic structure (a periodically varying thickness and/or periodically varying elastic and inertial properties) in one direction tangent to the undeformed shell midsurface $M$. Shells of this kind are termed uniperiodic. Moreover, it is assumed that the uniperiodic cylindrical shells, being objects of our considerations, are composed of a very large number of identical elements and every such element is treated as a shallow shell. It means that the period of inhomogeneity is very large compared with the maximum shell thickness and very small as compared to the midsurface curvature radius as well as the smallest characteristic length dimension of the shell midsurface in the periodicity direction. This uniperiodic structure of cylindrical shells considered here can be related to the periodically spaced dense system of ribs as shown in Figure 1.1.

For the uniperiodic cylindrical shells the known governing equations of the KirchhoffLove shell theory involve periodic highly oscillating and noncontinuous coefficients. Hence, in most cases direct application of these equations to analyze engineering problems in periodic shells is very complicated, particularly from the computational viewpoint. That is why the aim of this contribution was to propose a new nonasymptotic model of uniperiodic cylindrical shells for problems of dynamics and dynamical stability, which has constant coefficients in direction of periodicity and hence can be applied as a proper analytical tool for investigations of engineering problems in the shell under considerations. Moreover, the proposed model takes into account the effect of periodicity cell size on the global shell dynamics and dynamical stability as well as stationary stability, called the length-scale effect, which is neglected in the known homogenized models derived by asymptotic methods. 
In order to derive the model equations the tolerance averaging procedure given in [21], has been applied to governing equations of the Kirchhoff-Love second-order shell theory for thin linear-elastic cylindrical shells, that is, to (2.2)-(2.4). The proposed averaged model called the tolerance model of dynamic and dynamical stability problems for uniperiodic cylindrical shells is represented by a system of partial differential equations (4.8), (4.9) with coefficients which are constant in the direction of periodicity. The basic unknowns are the macrodisplacements $U_{\alpha}, W$ and the fluctuation variables $Q_{\alpha}^{A}, V^{A}, A=1,2, \ldots, N$, which have to be slowly-varying functions with respect to the cell and certain tolerance system. This requirement imposes certain restrictions on the class of problems described by the model under consideration. In order to obtain the governing equations the modeshape (shape) functions $h^{A}, g^{A}, A=1,2, \ldots, N$, should be derived from the periodic finite element method discretization of the cell or obtained as solutions to periodic eigenvalue problem on the cell given by (4.5). This eigenvalue problem describes free periodic vibrations of the cell, and hence the eigenfunctions $h^{A}, g^{A}, A=1,2, \ldots, N$, represent the expected forms of the oscillating part of free vibration modes of the periodicity cell. Moreover, in most problems the analysis is restricted to the simplest case $N=1$ in which we take into account only the lowest natural vibration modes (in directions tangent and normal to the shell midsurface) related to the smallest free vibration frequencies. Let us note that the model proposed here can be treated as a certain generalization of the models given in $[17,18]$.

The derived model has been used in this paper to investigate free vibrations of uniperiodically densely stringer-stiffened cylindrical shell. From the illustrative example it follows that in the framework of the nonasymptotic model proposed in this contribution, not only the fundamental lower but also the additional higher free vibration frequencies can be determined and analyzed. These additional higher free vibration frequencies depend on the period length and cannot be derived from the asymptotic models. Moreover, differences between values of fundamental lower free vibration frequencies derived from the tolerance model and those obtained from the asymptotic one are negligibly small. It means that the effect of the period length on the free vibrations of the considered shell can be neglected and hence, the homogenized (asymptotic) model is sufficient from the point of view of calculation for this dynamic problem.

Problems related to various applications of the proposed equations (4.7)-(4.9) to dynamics and dynamical stability of uniperiodic cylindrical shells and determination of the mode-shape functions from periodic eigenvalue problem given by (4.5) are reserved for a separate paper.

\section{Acknowledgment}

The paper was presented at DSTA '05 (Dynamical Systems Theory and Applications) conference held on Łódź, December 12-15, 2005.

\section{References}

[1] S. A. Ambartsumyan, The General Theory of Anisotropic Shells, Nauka, Moscow, 1974.

[2] I. Ja. Amiro and V. A. Zaruckiü, Theory of Ribbed Shells, Methods for Calculating Shells, vol. 2, Naukova Dumka, Kiev, 1980. 
[3] E. Baron, On dynamic stability of an uniperiodic medium thickness plate band, Journal of Theoretical and Applied Mechanics 41 (2003), 305-321.

[4] D. O. Brush and B. O. Almroth, Buckling of Bars, Plates and Shells, McGraw-Hill, New York, 1975.

[5] D. Caillerie, Thin elastic and periodic plates, Mathematical Methods in the Applied Sciences 6 (1984), no. 2, 159-191.

[6] G. D. Gavrylenko, Stability of Ribbed Cylindrical Shells in Nonuniform Stress-Strain State, Naukova Dumka, Kiev, 1989.

[7] I. Grigoliuk and V. V. Kabanov, The Shell Stability, Nauka, Moscow, 1978.

[8] J. Jędrysiak, On the stability of thin periodic plates, European Journal of Mechanics. A. Solids 19 (2000), no. 3, 487-502.

[9] R. V. Kohn and M. Vogelius, A new model for thin plates with rapidly varying thickness, International Journal of Solids and Structures 20 (1984), no. 4, 331-350.

[10] A. G. Kolpakov, Homogenized model for plate periodic structure with initial stresses, International Journal of Engineering Science 38 (2000), no. 18, 2079-2094.

[11] T. Lewiński and J. J. Telega, Asymptotic method of homogenization of two models of elastic shells, Archives of Mechanics 40 (1988), no. 5-6, 705-723 (1989).

[12] _ Plates, Laminates and Shells. Asymptotic Analysis and Homogenization, Series on Advances in Mathematics for Applied Sciences, vol. 52, World Scientific, New Jersey, 2000.

[13] A. Lutoborski, Homogenization of linear elastic shells, Journal of Elasticity 15 (1985), no. 1, 6987.

[14] B. Michalak, Stability of elastic slightly wrinkled plates, Acta Mechanica 130 (1998), no. 1-2, 111119.

[15] W. Nagórko and C. Woźniak, Nonasymptotic modelling of thin plates reinforced by a system of stiffeners, Electronic Journal of Polish Agricultural Universities, Series Civil Engineering 5 (2002), no. 2.

[16] W. Pietraszkiewicz, Geometrically nonlinear theories of thin elastic shells, Advances in Mechanics 12 (1989), no. 1, 51-130.

[17] B. Tomczyk, On the modelling of thin uniperiodic cylindrical shells, Journal of Theoretical and Applied Mechanics 41 (2003), no. 4, 755-774.

[18] - On stability of thin periodically densely stiffened cylindrical shells, Journal of Theoretical and Applied Mechanics 43 (2005), 427-455.

[19] T. von Kármán and H.-S. Tsien, The buckling of thin cylindrical shells under axial compression, Journal of the Aeronautical Sciences 8 (1941), 303-312.

[20] C. Woźniak, On dynamics of substructured shells, Journal of Theoretical and Applied Mechanics 37 (1999), 255-265.

[21] C. Woźniak and E. Wierzbicki, Averaging Techniques in Thermomechanics of Composite Solids, Wydawnictwo Politechniki Częstochowskiej, Czestochowa, 2000.

Barbara Tomczyk: Department of Structural Mechanics, Technical University of Łódź, Al. Politechniki 6, 90-924 Łódź, Poland

E-mail address: btomczyk@p.lodz.pl 


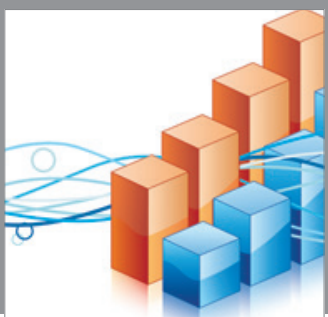

Advances in

Operations Research

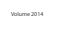

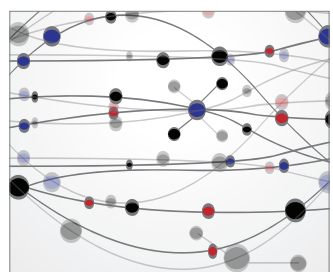

\section{The Scientific} World Journal
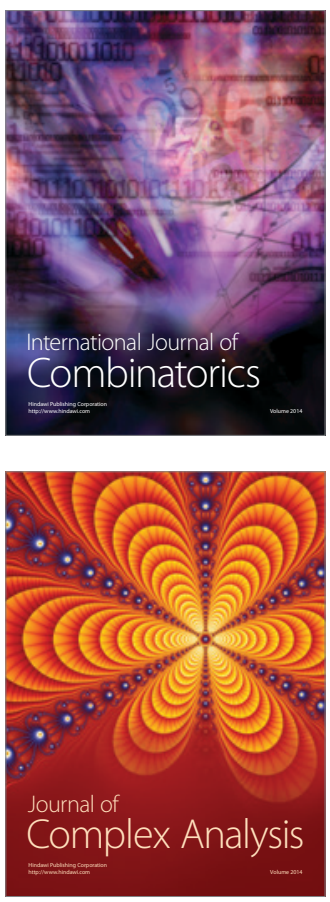

International Journal of

Mathematics and

Mathematical

Sciences
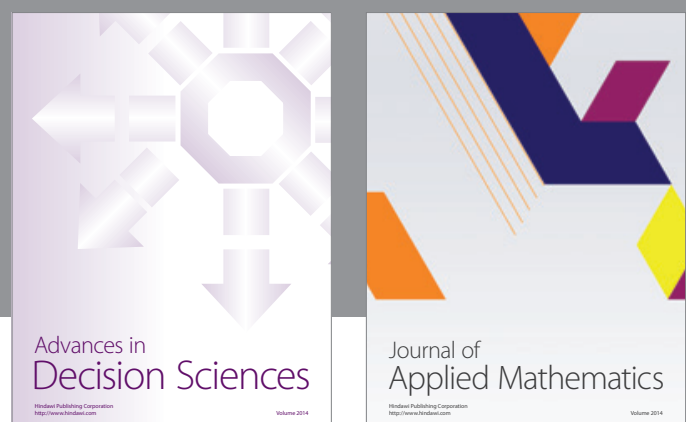

Journal of

Applied Mathematics
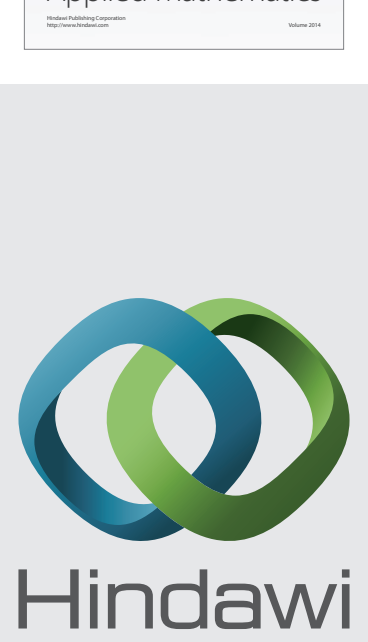

Submit your manuscripts at http://www.hindawi.com
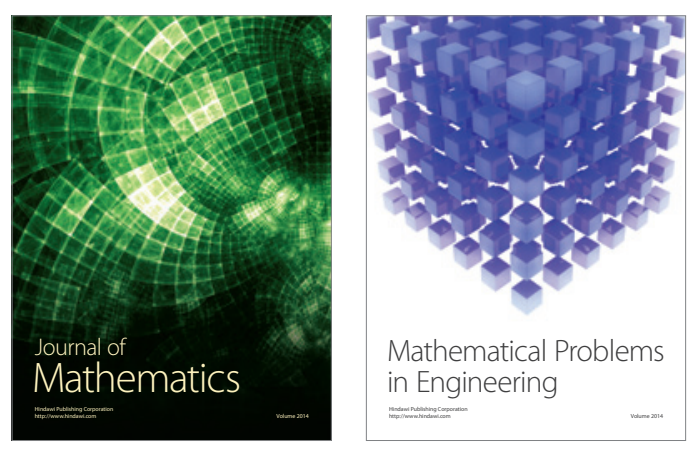

Mathematical Problems in Engineering
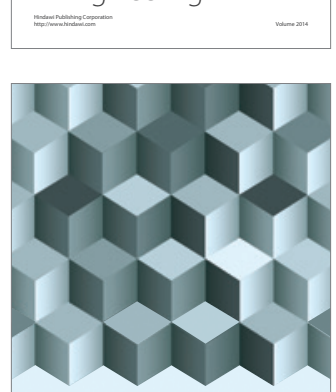

Journal of

Function Spaces
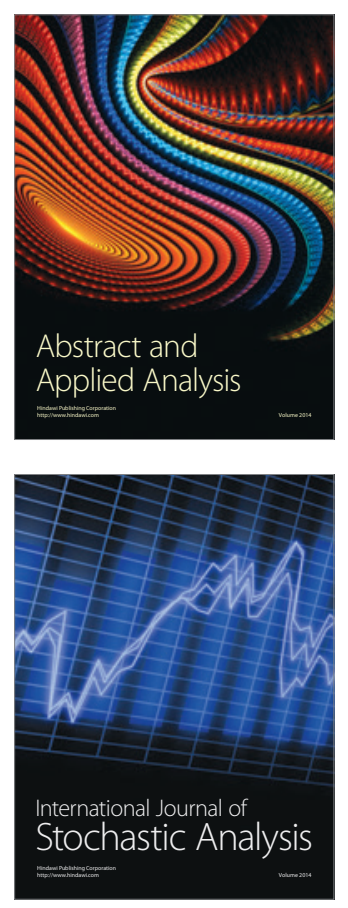

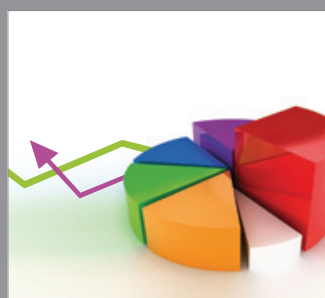

ournal of

Probability and Statistics

Promensencen
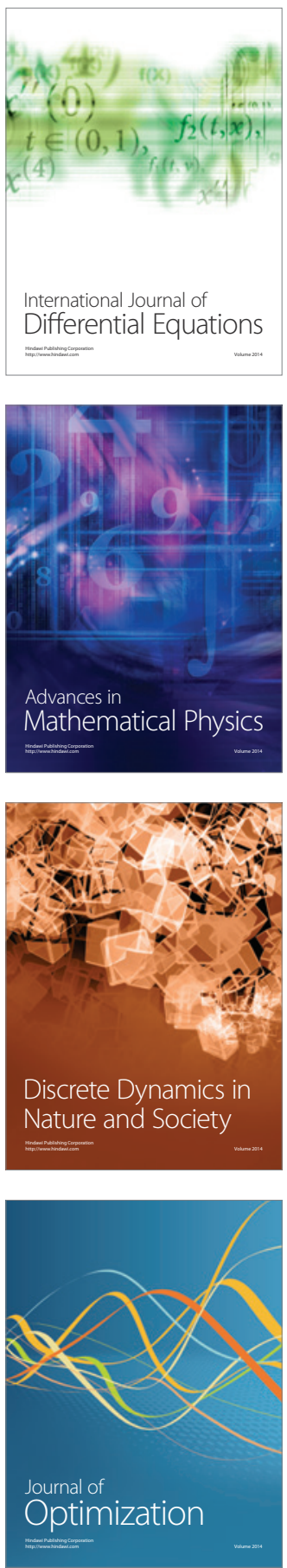